DOE/CE/40005

DOE/CE/40864

DOE/CE/40864-.TI-Pt.2

DEVELOPMENT OF LABORATORY AND PROCESS SENSORS TO MONITOR PARTICLE SIZE DISTRIBUTION

OF INDUSTRIAL SLURRIES

(Including Shape Characterization)

Final Technical Report

By:

Hemant P. Pendse, Principal Investigator

OCTOBER 1996

Work Performed Under Cooperative Agreements DE-FC05-88-CE40864 \& 94-CE40005

For:

U. S. Department of Energy,

Office of Industrial Technologies, Washington, D. C.

By

Pen Kem, Inc., Bedford Hills, NY 10507

P\&G Systems, Inc., Orono, ME 04473

MASTER

University of Maine, Orono, ME 04469

DISTRBBUTION OF THIS DOCUMENT IS UNMMTIED

APPROVED FOR RELEASE OR

PUBLCATION. INTEL. PROP. GP., OFC. OF CHIEF COUNSEL, DOEIORO by El Dellonde loli20198 


\section{DISCLAIMER}

This report was prepared as an account of work sponsored by an agency of the United States Government. Neither the United States Government nor any agency thereof, nor any of their employees, makes any warranty, express or implied, or assumes any legal liability or responsibility for the accuracy, completeness, or usefulness of any information, apparatus, product, or process disclosed, or represents that its use would not infringe privately owned rights. Reference herein to any specific commercial product, process, or service by trade name, trademark, manufacturer, or otherwise does not necessarily constitute or imply its endorsement, recom. mendation, or favoring by the United States Government or any agency thereof. The views and opinions of authors expressed herein do not necessarily state or reflect those of the United States Government or any agency thereof. 


\section{DISCLAIMER}

Portions of this document may be illegible in electronic image products. Images are produced from the best available original document. 
PSD Sensor Project including Shape Characterization

\title{
DEVELOPMENT OF LABORATORY AND PROCESS SENSORS TO MONITOR PARTICLE SIZE DISTRIBUTION OF INDUSTRIAL SLURRIES (Including Shape Characterization)
}

\author{
Final Technical Report
}

\author{
Hemant P. Pendse, Principal Investigator \\ Professor in Chemical Engineering and \\ Director, Industrial Process Control Sensor Systems (IPCoSS) Program, \\ University of Maine \\ and \\ Philip J. Goetz (President, Pen Kem, Inc.) \\ Arvind Sharma (Graduate Student, University of Maine) \\ Wei Han (Graduate Student, University of Maine) \\ Timothy C. Bliss (Graduate Student, University of Maine)
}

\begin{abstract}
The overall goal of the Particle Size Distribution (PSD) sensor projects was to develop and commercialize a sensor system capable of particle analysis, in terms of size distributions, using concentrated suspensions at high solids concentrations. The early research was focused on application of ultrasonic spectroscopy of inorganic pigment slurries (e. g. titanium dioxide) commonly encountered on paper industry. . During the project prototypes were tested in both academic and industrial laboratories. Work also involved successful field tests of the on-line prototype at a pigment manufacturing facility. Pen Kem continued the work at its cost beyond the initial funded period from March '92 to September '94. The first project (DE-FC05-88CE40684), which began in September 1988, culminated in a commercial laboratory instrument, Pen Kem AcoustoPhor ${ }^{\mathrm{TM}}$ 8000, put on the market in June 1993.

The follow-on project was aimed at investigation of shape and orientation effects on ultrasonic spectroscopy. A new cooperative agreement was awarded in September 1994 (DE-FC05-94CE40005) to develop shape characterization capabilities deemed critical by the clay industry. This follow-on project achieved following successes: A theoretical model was developed to account for the effects of size-dependent aspect ratios of spheroid particles under different orientations on ultrasound attenuation spectra of concentrated slurries. The theoretical model was confirmed by laboratory tests on kaolin slurries. An algorithm was developed to simulate evolution of particle orientation fields in simple squeezing flows.
\end{abstract}




\section{PSD Sensor Project including Shape Characterization}

Information on orientation field evolution associated with squeezing flows can be used to infer shape factors from attenuation spectra obtained in the continuously-changing-gap mode, as compared to those in the multiple-gap-sequence mode. Active spectroscopy, which involves on-the-fly attenuation measurements in the continuously-changing-gap mode, thus can provide a tool for shape analysis in concentrated slurries. Incorporation of limited shape analysis capabilities in the present commercial instrument appears to be feasible even without significant hardware modifications.

The overall program meets the industrial needs of many pigment (TiO2, Clay, and Calcium Carbonate) suppliers to the paper industry. A sensor for monitoring size and shape of pigments in slurry processing area without dilution can have substantial impact on energy savings through reduction of over grinding and thermal drying loads $(0.034$ quads/year). Also such a sensor can lead to significant reduction in the substandard product fraction that would need rework. Availability of this sensor for industrial QC as well as for process control is expected to lead to new methods and techniques for developing engineered slurry products thus enhancing industry competitiveness. Rework cost reduction can be several million dollars per year. Current commercial laboratory instrument, Pen Kem AcoustoPhor ${ }^{\mathrm{TM}} 8000$ has already began to make impact in pigment processing industry. 


\section{Table of Contents}

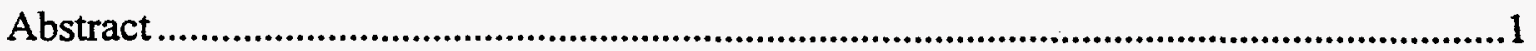

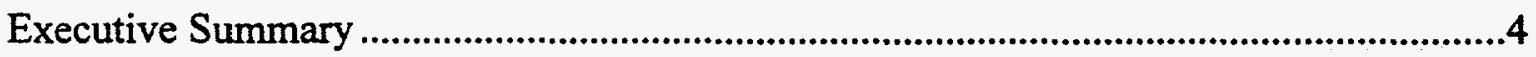

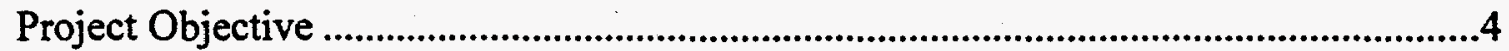

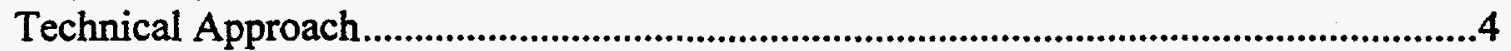

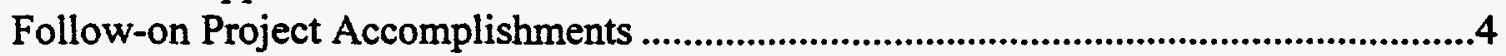

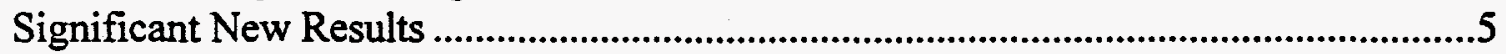

I. PSD Sensor: Commercialization of AcoustoPhor 8000 ....................................................6

Section Summary...............................................................................................................6

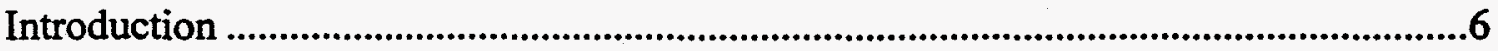

Commercialization Efforts ............................................................................................

II. Background on Ultrasonic Spectroscopy for PSD Analysis ..........................................15

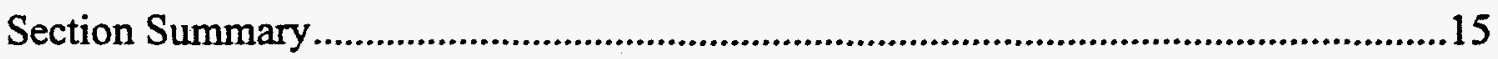

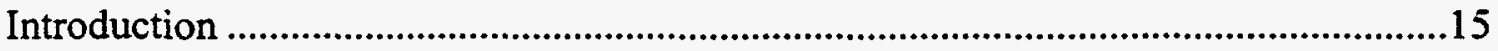

Recent Activity in Instrumentation for Ultrasound Spectroscopy...................................16

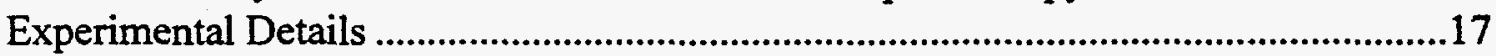

Illustrative Results ..........................................................................................................18

Discussion................................................................................................................19

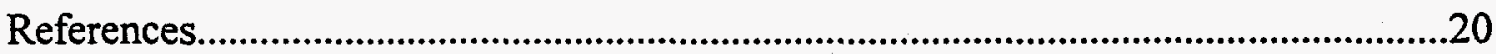

III. Analysis of Shape Effects: Tests using Clay Slurries ..................................................25

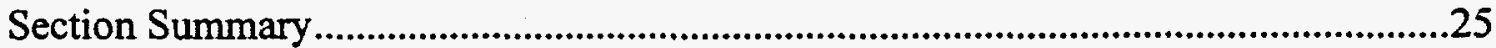

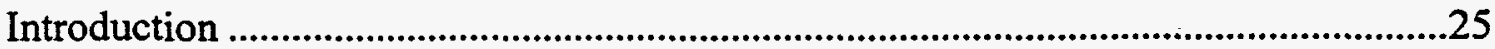

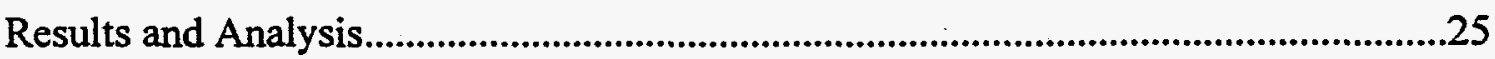

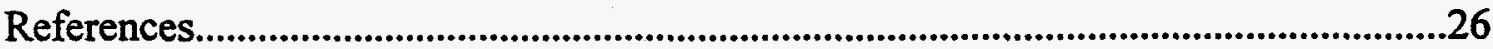

IV. Particle Orientation Evolution in Suspensions subjected to Squeezing Flow ..............29

Section Summary............................................................................................................29

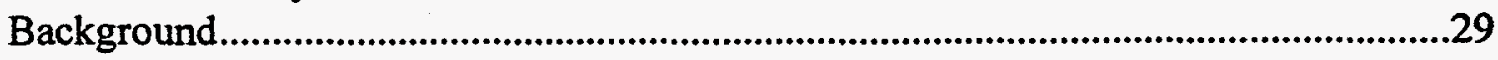

Illustrative Results for oblate and prolate orientation evolution ........................................30

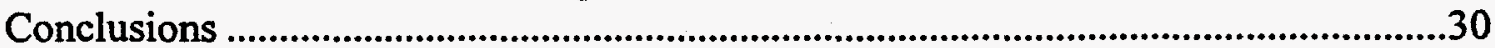

List of Published Technical Papers resulting from the PSD Sensor project.......................34

List of Ph. D. Dissertations resulting from the PSD Sensor Project......................................34

List of M. S. Theses resulting from the PSD Sensor Project....................................................34

Interim Technical Report on PSD Sensor Project (October 1992) ......................................35

$\begin{array}{ll}\text { Final Technical Report (October 1996) } & \text { Page } 3\end{array}$ 
PSD Sensor Project including Shape Characterization

\section{Executive Summary}

\section{Project Objective}

The particle size distribution (PSD) sensor development project was aimed at providing a sensor system capable of monitoring PSD of high solids inorganic pigment slurries under process conditions without dilution. The research culminated in a commercial laboratory instrument. The follow-on 'Shape Analysis' part of the PSD Sensor Project was focused on enhancing recently developed PSD Sensor System by adding shape analysis capabilities to increase industrial acceptance of the new technology. One of the target application involves process monitoring in delamination of clay.

\section{Technical Approach}

The PSD sensor uses a programmable ultrasonic spectrometer to obtain attenuation coefficient spectra of test slurries over a wide range of frequencies as described in the 1992 Interim Technical Report (unpublished, a copy is attached). Experimental details of the measurement technique are later discussed in detail in Sharma's Ph. D. dissertation (220pp.) published in December 1995. The PSD analysis used in this instrument is based on theoretical models developed for concentrated suspensions of spherical particles, which has been well documented in Strout's Ph. D. dissertation (151 pp.) published in May 1991. On the other hand, the shape characterization is based on interpretation of ultrasound response of concentrated suspensions using theoretical predictions of attenuation spectra for suspensions containing spheroidal particles. Such theoretical predictions are based on a model that takes into account the effects of particle size, aspect ratio and orientation over a wide ranges of frequencies and particle concentrations. The general theoretical model that takes into effects of particle shape and orientation is discussed in detail in Han's Ph. D. dissertation (262 pp.) published in December 1995.

\section{Follow-on Project Accomplishments}

1. A theoretical model (Pendse-Han Model) to predict attenuation spectra of concentrated suspensions of high density-contrast, non-spherical particles (spheroid) that takes into account volume equivalent diameter, aspect ratio, orientation, volume fraction and operating frequency was developed. A test program was installed at Pen Kem in the Summer of 1995.

2. Experimental attenuation spectra for 11 kaolin slurries (with particle concentrations ranging from $0.6 \%$ to $35 \%$ by weight at 18 frequencies over 3 to $100 \mathrm{MHz}$ range) were obtained using the on-line prototype of AcoustoPhor 8000 . These data were obtained with the recirculating cross-flow on i.e. in a flow-though mode.

3. The kaolin slurry data were analyzed using the Pendse-Han model, assuming random orientations for particles in the sensing zone, to assess sensitivity to shape factors. A oneday workshop was held at Pen Kem to discuss theoretical models, experimental data, and analysis. See Chapter 5 of Han's dissertation for details. 
PSD Sensor Project including Shape Characterization

4. A procedure (Pendse-Bliss Algorithm) was developed to simulate evolution of particle orientation fields for a simple squeezing flow geometry, using the Transversely Isotropic Fluid (TIF) model. This development is documented Bliss's Master's Thesis (95 pp.), completed in August 1996, which is available from the University of Maine library.

5. Methodologies to infer shape characteristics were developed based on (a) attenuation spectra obtained with a cross-flow using on-axis transducer configuration, and (b) attenuation spectra obtained in a stop-flow mode using off-axis transducer configuration while sensor gap decreases continuously.

6. Hardware modifications were designed for (a) transducer mounting in the sleeves and (b) sleeve mounting in the chamber, necessary to use a continuously decreasing gap mode with an off-axis transducer configuration.

\section{Significant New Results}

1. Attenuation spectra of clay slurries are expected to be sensitive to changes the particle aspect ratio at high frequencies and high particle concentrations. Data at low frequencies (less than $10 \mathrm{MHz}$ ) or at low concentrations ( 5 volume percent) will be of limited use for shape analysis.

2. Attenuation spectra under (a) random orientations and (b) parallel orientations are expected to be significantly different from each other.

3. Expected orientation fields under squeezing flow are non-uniform and transient, that is position and time dependent. Off-axis line-of-sight measurements will be needed rather than on-axis line-of-sight measurements.

4. Off-axis transducer configuration will involve additional alignment of sleeves around their common axis to make the opposing transducers become co-linear. If the present transducers are to be used in the modified chamber the sleeve diameter and the overall chamber size will be too large. If no change in the sleeve diameter and the overall chamber size is desired, then new custom traducers with smaller case diameter $(8 \mathrm{~mm})$ will have to be used.

5. The modifications in the electrical and mechanical subsystems necessary to implement the 'active spectroscopy' (i.e. on-the-fly measurements with a continuously changing gap) were found to be quite complex. Such complex hardware modifications need to be phased in at appropriate times later during the normal product development cycle of the commercial AcoustoPhor 8000System.

6. Incorporation of limited shape analysis capabilities in the present commercial instrument appears to be feasible, even without significant hardware modifications. 


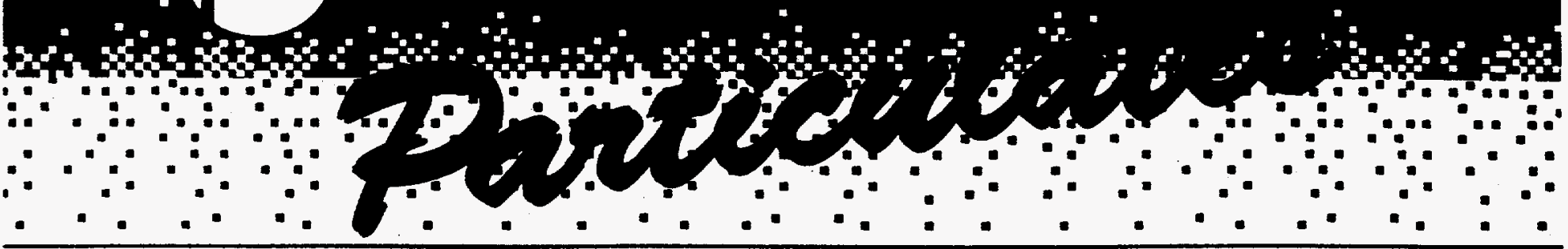

THE NEWSLETTER OF COLLOIO TECHNOLOGY - SUMMER 1993

\section{P R E S I D E N T Scanuen}

\section{Our 21st Anniversary Newsletter}

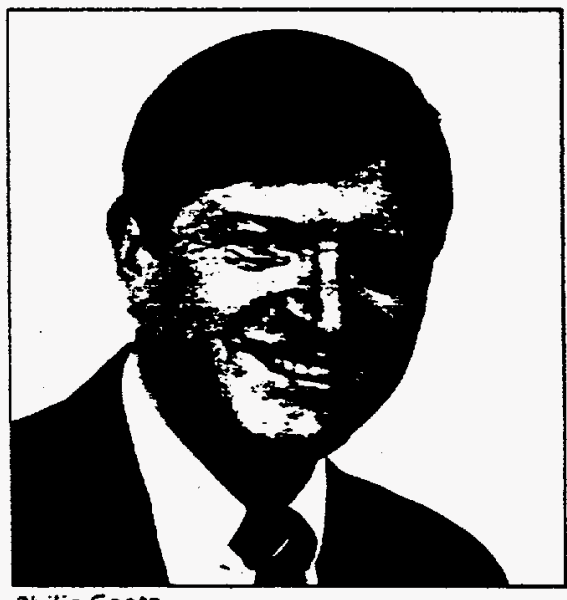

Philip Goetz

It is hard to believe that twenty one years have gone by since founding Pen Kem. In this Newsletter called "Particulates" we want to tell you something about Pen Kem's history as well as our plans for the future.

Each section of Particulates is aimed at meeting the needs of the colloid community. The User Round Table focuses on items of interest to our current customers. In the months ahead we will be working with various users to explore common problems and opportunities. Application Highlights will give you some feeling for the breadth of the work in our Applications Lab. The Guest Interview features commentary of interest to the Pen Kem community of customers and friends.

\section{The good old days}

Before starting Pen Kem in 1972. I had already spent twelve years as an electrical engineer in the aerospace industry developing various high tech military products such as doppler radar navigation systems for military aircraft. I guess you might say
I was in the vanguard of people leaving the military to search for a career in the commercial sector.

I still remember designing Pen Kem's first zeta potential instrument on my dining room table. Nine months later we delivered the first Lazer Zee Meter to the Environmental Protection Agency's Water Hygiene Division to study problems related to asbestos fibers in municipal water supplies. Today the Lazer Zee has become a world standard with almost one thousand units installed in over fifty countries around the world.

I have been exhibiting at the Pittsburgh Conference for almost twenty years. In the beginning, common questions asked by visitors to our booth were "What is a colloid?" or "What is zeta potential?". Now everyone seems to know what a colloid is. Also, the importance of electrokinetic phenomena is more widely appreciated, if not always completely understood.

\section{The times they are a'changing}

It is encouraging to see how the awareness of colloid science has grown over the past two decades. I like to think that Pen Kem had something to do with popularizing the role of colloid science and technology.

Pen Kem has changed dramatically over the years, as well as the colloid marketplace in general. A key area of change is the complexity of modern instruments. The Lazer Zee Meter was designed by a șingle person on a dining room table, whereas our new AcoustoPhor 8000 required a multi- disciplinary team including the federal government, industry sponsorship and strong academic support.

The AcoustoPhor 8000 was supported by a half-million dollar grant from the US Department of Energy. Collaboration and support from the Pigments Division of DuPont helped ensure that the final product will meet the needs of the chemical industry. A strong academic team led by Professor Hemant Pendse at the University of Maine provided a sound theoretical basis for the design.

\section{Full steam ahead}

This trend toward increased complexity in instrumentation design will continue. In the next issue of Particulates, we will tell you more about how Pen Kem plans to meet this challenge.

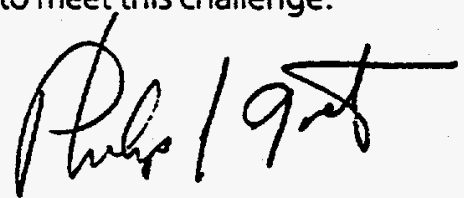

\section{Table of Contents}

Presidents Corner

- User Round Table

口 Applicaton Highligh

- Cucst Interview

- New Product focur

a Colloid Quips

- Calendar 
PSD Sensor Project including Shape Characterization

\title{
I. PSD Sensor: Commercialization of AcoustoPhor 8000
}

\author{
Philip J. Goetz, Pen Kem, Inc., Bedford Hills, NY 10507
}

\section{Section Summary}

This section deals with the commercialization status of the Pen Kem AcoustoPhor ${ }^{\mathrm{TM}}$ 8000 , an instrument for determining the particle size distribution (PSD) in concentrated slurries. The PSD sensor development was supported by the Department of Energy (DOE) and has been successfully employed in a commercial instrument serving the colloid science community. The on-line prototype was evaluated in a pigment manufacturing plant. Continued development at Pen Kem, following the DOE funded effort, has dramatically improved the measurements, prediction, size distribution estimation, and provided a versatile user interface. Several presentations have been given at technical meetings and trade shows. Application laboratories have been set up in Bedford Hill, New York and Tokyo, Japan.

\section{Introduction}

\section{Scientific Overview}

The AcoustoPhor ${ }^{\mathrm{TM}} 8000$ technology can be broken down into four key areas: measurement, prediction, optimization, and the user interface. In more detail, the instrument measures the attenuation of sound over a frequency range from 1 to 100 $\mathrm{MHz}$. It must be able to predict the attenuation that would be experienced by a slurry having a given particle size distribution. Using the measured data and this prediction capability, the AcoustoPhor ${ }^{\mathrm{TM}}$ must then perform an optimization in which the estimated PSD solution is varied until the error between the measured attenuation and the predicted attenuation is minimized. Finally the instrument must provide a user interface which allows one to define the sample, define the experiment protocol, save the measured data, retrieve selected data as needed, and present the data in graphical or tabular form as required.

\section{Measurement of attenuation}

The attenuation of sound varies over a wide dynamic range. Plain water at a frequency of $1 \mathrm{MHz}$ has almost no attenuation, whereas at $100 \mathrm{MHz}$ even water has an attenuation of about $20 \mathrm{~dB} / \mathrm{cm}$. An attenuation of $20 \mathrm{~dB} / \mathrm{cm}$ means that the sound is attenuated by a power ratio of 100:1 over a distance of $1 \mathrm{~cm}$. In contrast, the attenuation of a $76 \mathrm{wt}$. \% $\mathrm{TiO}_{2}$ slurry can exceed $1000 \mathrm{~dB} / \mathrm{cm}$ at $100 \mathrm{MHz}$. This equals a power loss of $10^{100}: 1$ for each $\mathrm{cm}$ the sound wave penetrates through the slurry.

To give some feeling for the magnitude of this attenuation, consider the following hypothetical experiment. Grand Coulee Dam generates an output power of about $10^{10}$ watts. If it was possible to convert the output of Grand Coulee dam into ultrasonic power at $100 \mathrm{MHz}$, and then to launch this huge amount of power into this $76 \mathrm{wt}$. \% 
TiO2 slurry, the power remaining after traveling a distance of just $1 \mathrm{~mm}$ would not be enough, if converted back to electrical energy, to power a 1 watt flashlight. Furthermore it would take $10^{10}$ Grand Coulee Dams to transmit enough power to light this same flashlight if the sound was required to travel 10 times further; i.e. a distance of just $1 \mathrm{~cm}$ ! Of course, such experiments are impossible; the power would instantly vaporize our sample. From this example, it is clear that measuring acoustic attenuation from $0 \mathrm{~dB} / \mathrm{cm}$ to $1000 \mathrm{~dB} / \mathrm{cm}$ is a challenge, nevertheless the AcoustoPhor ${ }^{\mathrm{TM}} 8000$ has solved this problem.

\section{Various Attenuation Mechanisms}

Determination of the PSD by means of acoustic spectroscopy is similar, in some respects, to quasi elastic light scattering (QELS). However, in acoustics spectroscopy, scattering is just one of several mechanisms by which the sound may be attenuated. There are at least five separate loss mechanisms which can produce acoustic attenuation, namely: scattering, viscous, thermal, structural, and electrokinetic losses.

Scattering loss occur when some of the acoustic energy is scattered out of the direct path between the transmitting and recovery transducers by sufficiently large particles. Scattering losses increase with increasing frequency but are often negligible for submicron particles below $100 \mathrm{MHz}$.

Viscous losses occur when some of the acoustic energy is converted to heat as a result of a motion of the particles relative to the surrounding viscous fluid. The effect is similar to the energy lost by a sedimenting particle as its potential energy is converted to heat as it settles. This relative motion depends of the inertia of the particle. The acoustic attenuation spectra due to such viscous losses exhibits a bell shaped spectra, and the maximum attenuation occurs at a critical frequency related to the particle diameter. As the density contrast between the particles and the media goes to zero, viscous losses disappear.

Thermal losses occur when the particles are not rigid and have different thermodynamic properties than the suspending media. The volume of the particle changes with the sound pressure amplitude, the temperature of the particle fluctuates accordingly, and sound energy is again converted to heat during each cycle of the sound wave.

Structural losses occur when acoustic energy is converted to heat in deforming or flexing whatever structure connects the particulates in the system. These structural losses are not yet very well understood, but can sometimes play a significant role.

Finally, Electrokinetic losses result from interaction between the particle's double layer surrounding the particles and the sound field. These losses are generally negligible and will not be discussed here further. 
At the present time our prediction model accounts precisely for the viscous losses in concentrated polydisperse systems. Our current model is therefore sufficient to describe submicron slurries of relatively high density contrast materials such as inorganic pigments, ceramics, and clays at concentrations up to 50 volume \%. However, the theory for scattering losses should be extended to include the effects of both polydispersity and concentration effects if we want to improve the psd interpretation of slurries with particles much larger than say 1 micron. Similarly, the theory for thermal losses must also be extended to include polydispersity and concentration in order to improve the psd interpretation of such non-rigid low density contrast systems as emulsions, lattices, food products, etc. Because of these theoretical limitations, we have chosen to market the product initially in the ceramics and pigments area while at the same time developing some experience in other areas.

\section{Commercialization Efforts}

At the conclusion of the DOE funded program, done in collaboration with University of Maine, the design was far from a commercially viable product. Very significant work was required in all four areas described above, namely: measurement, prediction, optimization, and the user interface. This commercialization work was funded by Pen Kem, and accomplished in Bedford Hills exclusively by Pen Kem personnel. Dr. Andrei Dukhin, formerly of the Ukrainian Academy of Science, now Technical Director of Pen Kem, played a key role in these commercialization efforts.

Improving measurement of acoustic spectra

Pen Kem's commercialization efforts aimed at improving the measurement can be broken down into two main areas of activity: packaging and cost reduction.

\section{Packaging}

A block diagram of the AcoustoPhor ${ }^{\mathrm{TM}} 8000$ system is shown in Figure 1. A peristaltic pump aspirates sample at a controlled rate from the sample cup into the test loop. Once the sample is loaded, the sample valve is put in the "circulate" position such that the test sample is continuously circulated within the test loop. Probes measure the temperature, $\mathrm{pH}$, and conductivity of the test sample. An injection port allows reagents to be added to the test sample from a digital burette under computer control. Transmit and receive ultrasonic transducer are placed so that the test sample flows between them. The gap between these transducers is controlled by a stepping motor and can be set from roughly 100 microns to $25 \mathrm{~mm}$ under computer control. Bursts of RF energy at frequencies from 1 to $100 \mathrm{MHz}$ are transmitted and then received a short time later after transiting the slurry. The slope of the received signal vs. the gap between the transducers gives us the attenuation rate as expressed in $\mathrm{dB} / \mathrm{cm}$. After measurement, the contents of the test loop is displaced by pressurized air and is either returned to the sample cup or discharged to the drain. The test loop is then automatically cleaned.

The commercial package is shown in Figure 2. The user simply installs a cup with the sample, defines the sample, selects a measurement protocol, and the rest is automatic. 
The sample is loaded, chemicals added as needed, the attenuation measured, the sample disposed of and the system cleaned.

\section{Cost Reduction}

In order to be competitive with other PSD techniques, a basic laboratory version of the system should sell for about $\$ 60,000$. Such high performance acoustic spectroscopy equipment is unavoidably complex in both hardware and software. Where we have had a choice, we have opted to make the software more complex in exchange for simplifications in the hardware. Computer technology becomes cheaper each year while traditional hardware components, and the labor to assemble them, becomes more expensive.

However, we were careful during the design phase to partition the hardware/software tasks so as to take advantage of what we envisioned as emerging technology. Two areas of particular importance were the rapid growth of cellular phone technology and the -availability of inexpensive Field Programmable Gate Arrays (FPGA).

For example, one key part in the original design cost $\$ 7,000$ in 1990 , was replaced by a $\$ 700$ part in the Phase 2 program, and during the commercialization program is being replaced again by a $\$ 7$ part developed just in the past few months for the cellular phone market. Similarly, a $\$ 600$ synthesizer module occupying more than 20 sq. in. of printed circuit board space is being replaced by 5 integrated chips at a fraction of the cost and circuit board area.

Such improvements, plus the use of FPGA have allowed us to reduce the Receiver from four bulky modules interconnected by more than a dozen coax and ribbon cables to a single card that plugs into a single card slot in a standard high performance personal computer. This effort has not only reduced cost but significantly increased reliability and ease of manufacture.

\section{Sample handling}

Pen Kem's first customer for the AcoustoPhor ${ }^{\mathrm{TM}}$ was the largest surfactant manufacturer in Japan who wanted to measure the PSD of cement slurries at concentrations up to 76 wt. \%. Cement samples created some unique problems: plugging of valves and fittings, rapid abrasive degradation of fluid seals, and a sample which literally hardened to rock if inadvertently left in the chamber of more than an hour. To achieve reliable operation with cement samples we had to design custom valves and write automated cleaning procedures. Figure 3 shows a series of attenuation curves for cement samples over a wide range of concentration up to the target value of $76 \mathrm{wt} \%$, the concentration at which most cement is actually used in the field. A PSD for one of the cement samples is shown in Figure 4.

A major advantage of the acoustic technique is the ability to measure concentrates without dilution or sample preparation. In contrast, most conventional particle size 
instruments require dilution in some artificial media and then dispersion with a high energy ultrasonic probe prior to measurement. Sometimes it is also desirable to disperse concentrated samples prior to measurement with the AcoustoPhor ${ }^{\mathrm{TM}}$. Some samples may be time dependent because of flocculation or aggregation. In order to establish a baseline from which a time dependent PSD can be measured, we have installed a $100 \mathrm{~W}$ ultrasonic probe which, under software control, can re-disperse the sample prior to measurement.

Because samples such as coal slurries may be quite hydrophobic and not easily wetted, they may contain significant amount of air. This air may effect the attenuation spectra. Since we are not usually interested in characterizing the PSD of the entrained air, the sample handling syhstem was modified to provide, under software control, a vacuum to remove entrained air.

The initial on-line system measured a flowing sample at whatever process temperature was currently being used. For the laboratory unit, we wanted to control a temperature set point or alternatively, under software control, set up a controlled temperature profile. A Peltier device was installed in the test loop to provide automatic temperature control over the range of 20 to 50 degrees Centigrade.

The PSD depends on various processes which in most cases are sensitive to chemical modification of the particle surface. Therefore we provided digital burettes so as to have means to control the addition of various chemicals to the test sample as it was being measured. Figure 5 shows a sequence of PSD's for a single TiO2 sample with increasing doses of a surfactant.

\section{Prediction}

The prediction software computes an attenuation spectra, given a particle size distribution. The initial prediction software, developed during the DOE funded portion of the program by Hemant Pendse and co-workers at UMO, made certain simplifying assumptions to facilitate the analysis. For example, we assumed that the total attenuation could be represented as a linear superposition of the contribution of each individual size fraction. Furthermore, we assumed that the slurry consisted of only one disperse phase, e.g. alumina or silica, but not a mixture of both.

During the Pen Kem funded commercialization effort, additional theoretical work was accomplished by Dr. Dukhin at Pen Kem which has eliminated the need for this superposition assumption as well as the restriction on a single disperse phase. Dr. Dukhin is now documenting this work in a series of three technical papers which will be published in the Journal of Colloid and Interface Science during 1995.

PSD Estimation using Optimization Techniques

Optimization is the inverse of prediction. Prediction computes attenuation given a particle size distribution. Normally we do not know the PSD; we want to determine it! 
We can, however, measure attenuation. Optimization is the process by which we select a predicted PSD which yields the best match between the predicted attenuation and the actual measured attenuation.

The initial prediction software, developed during the DOE funded portion of the program by Hemant Pendse and co-workers at UMO, was suitable for the on-line application in which the sample being measured did not differ too much from measurement to measurement. It required a number of user selected parameters, which were obtained based on experience with a particular sample. It was not robust in the sense that it sometimes produced wrong and misleading PSD results if approximate values for the PSD were not specified by the user. In addition it was not possible to obtain an accurate PSD on fairly monodisperse samples. Furthermore, it was not possible to measure particles below 0.1 micron. Finally, it was impossible to work with disperse systems which consisted of a mixture disperse phases having different characteristics.

Each of these limitations in the Optimization software was addressed during the Pen Kem funded commercialization effort in Pen Kem's laboratory in Bedford Hills by Dr. Dukhin and co-workers. The original optimization used numerical inversion techniques, which are computationally quite efficient but have numerous well known shortcomings. The advent of faster low cost computers such as the Intel Pentium allowed us to consider a new approach to the optimization problem which is now the subject of a patent application. The success of this new method can be judged by means of the figures given below.

Figure 6 illustrates the robustness of the AcoustoPhor ${ }^{\mathrm{TM}}$ as measured by its ability to measure PSD's over a wide range of samples without the need for the user to set any adjustable parameters. It also illustrates the ability to characterize samples which are relatively monodisperse.

Figure 7 illustrates the ability to measure particles well below $0.1 \mathrm{um}$. The fluctuation about the linear regression line was less than 0.003 microns suggesting that the instrument may be very useful for tracking nucleation processes in a variety of applications.

Figure 8 illustrates the ability to measure a heterogeneous system consisting in this case of a mixture of both alumina and zirconia. This is a typical mixture used in many ceramic slips.

The User Interface

The user interface can be subdivided into three areas: 1.) means for the user to define the sample and the experiment to be performed, 2.) means to retrieve the desired data, and 3.) means to display the data in a meaningful way. 
During the DOE funded activity the user interface was essentially ignored. On-line measurements were done on the same process sample again and again so that little input was required from user. Each measurement was stored in a new data file and it was left to the user to catalog and retrieve the information. The system used only DOS, had no Windows interface, and only the most primitive graphics.

During the $\mathrm{pK}$ funded commercialization phase, a Windows user interface was created by the Pen Kem staff in Bedford Hills. The progress in this area was greatly aided by very productive software tools which have just recently become available. Development of such a Windows based program in the past has typically required many man years of effort. The availability of Microsoft Visual Basic 3.0, the Microsoft Access data base program and dynamic link library programs for graphics collapsed this software activity into a more manageable task. The present AcoustoPhor ${ }^{\mathrm{TM}}$ user interface incorporates the following features:

Sample Definition Dialog box for defining the sample characteristics such as the name of the material which composes the disperse phase, the weight fraction of the sample, etc. The entry of such data is aided by a Materials Library which contains the required physical properties for a large number of material such as alumina, silica, etc.

Experiment Protocol Dialog box for defining the experiment to be performed including such items as temperature profiles, chemical addition, etc.

Data base for storing all sample definitions, experiment protocols, measured attenuation data, calculated PSD results, etc. The user can retrieve selected information by specifying a "query" which defines such retrieval criteria as for example the date of measurement, nature of sample, $\mathrm{pH}$, temperature, etc.

$\square$ Graphics application which gives user a gallery of different types of presentations to select from.

Expert system for trouble shooting the hardware.

Help File to provide instructions on how to use the equipment. 
PSD Sensor Project including Shape Characterization
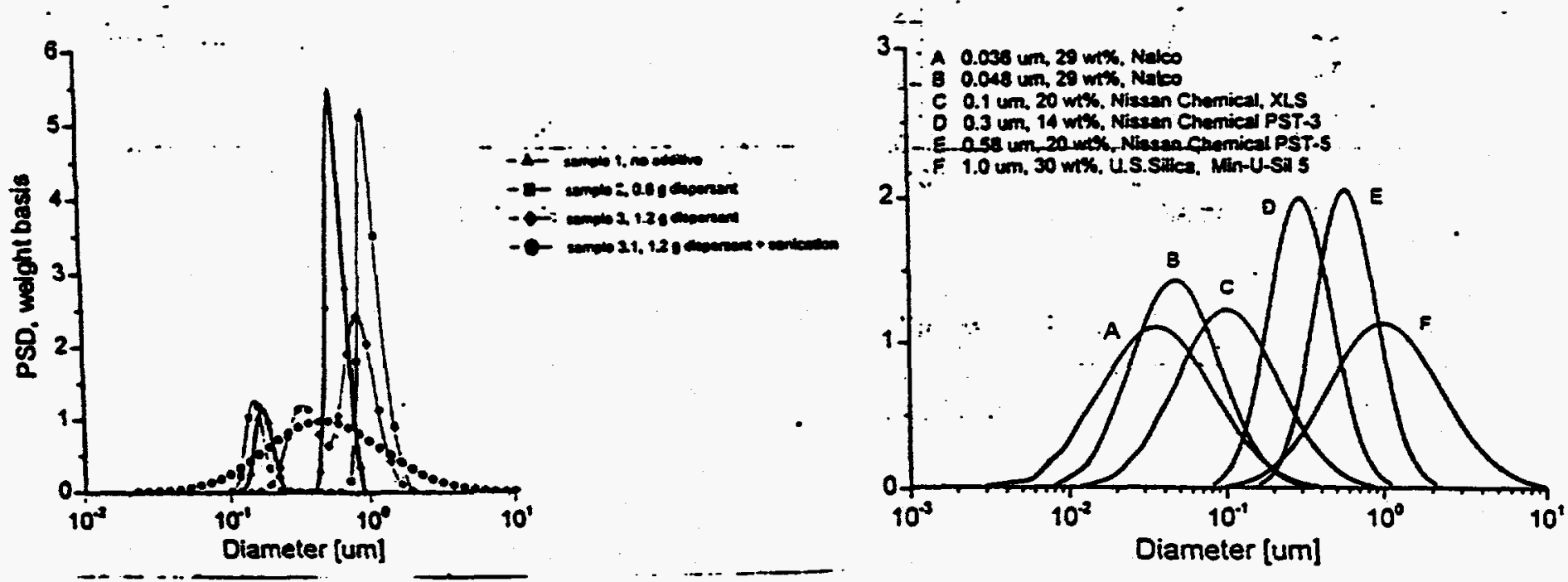

Figure 5 Effect of various doses of surfactant on PSD of $40 \mathrm{wt} . \% \mathrm{TiO}_{2}$ slurry.

Figure 6 PSD's for series of well dispersed silica samples.
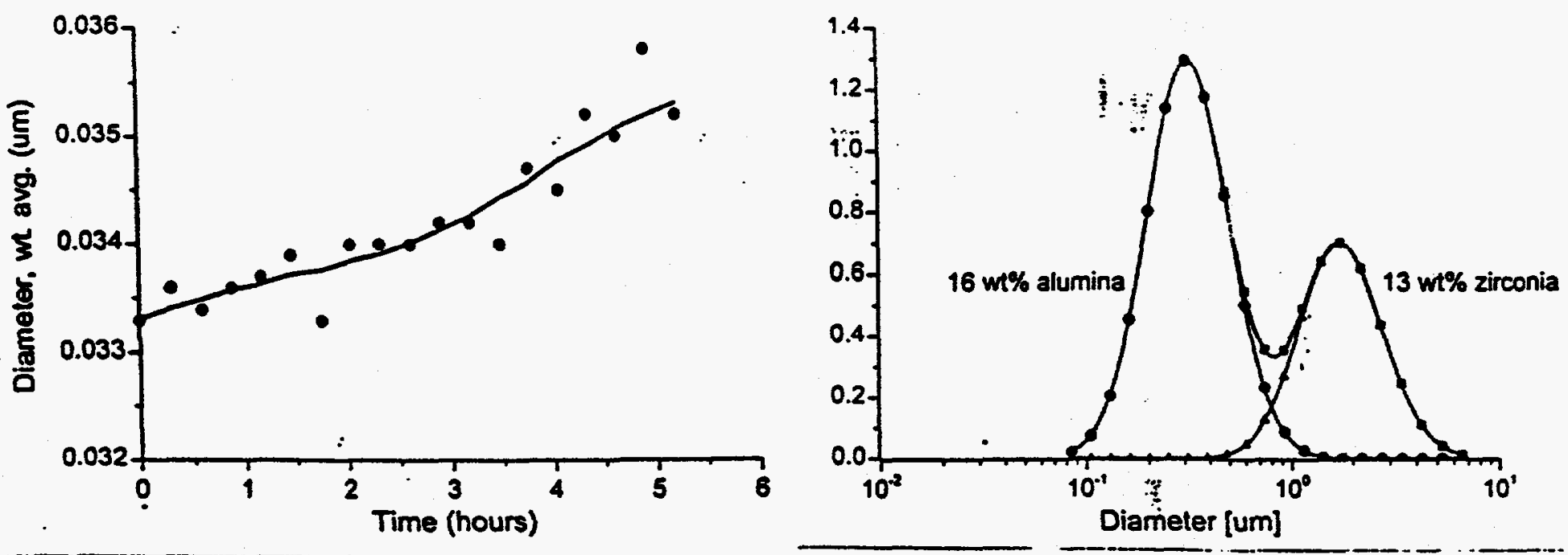

Figure 7 Mean particle size of nanosized colloidal silica as function of time.

Figure 8 PSD of 29 wt \% slurry consisting of mixture of both Alumina and Zirconia. 
PSD Sensor Project including Shape Characterization

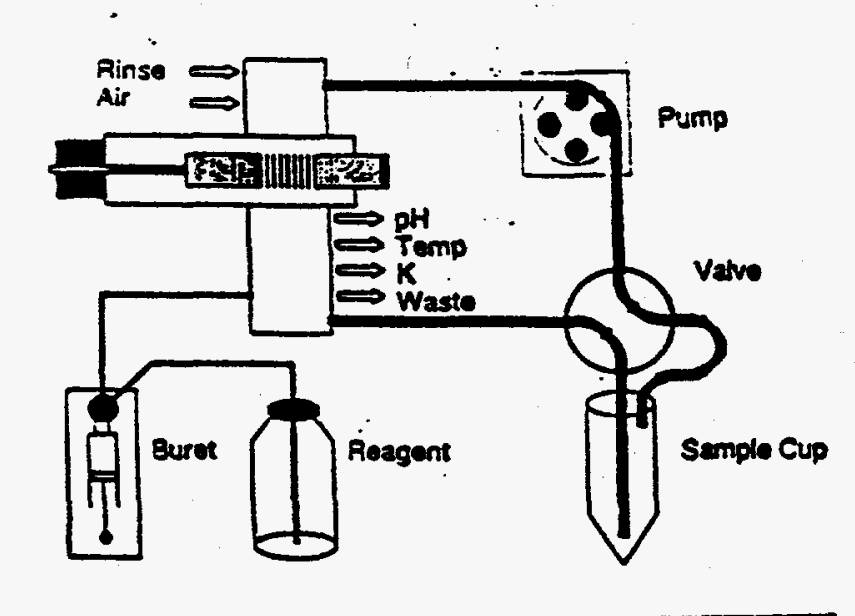

Figure 1 Block Diagram of Pen Kem AcoustoPhor ${ }^{\text {TI }} 8000$

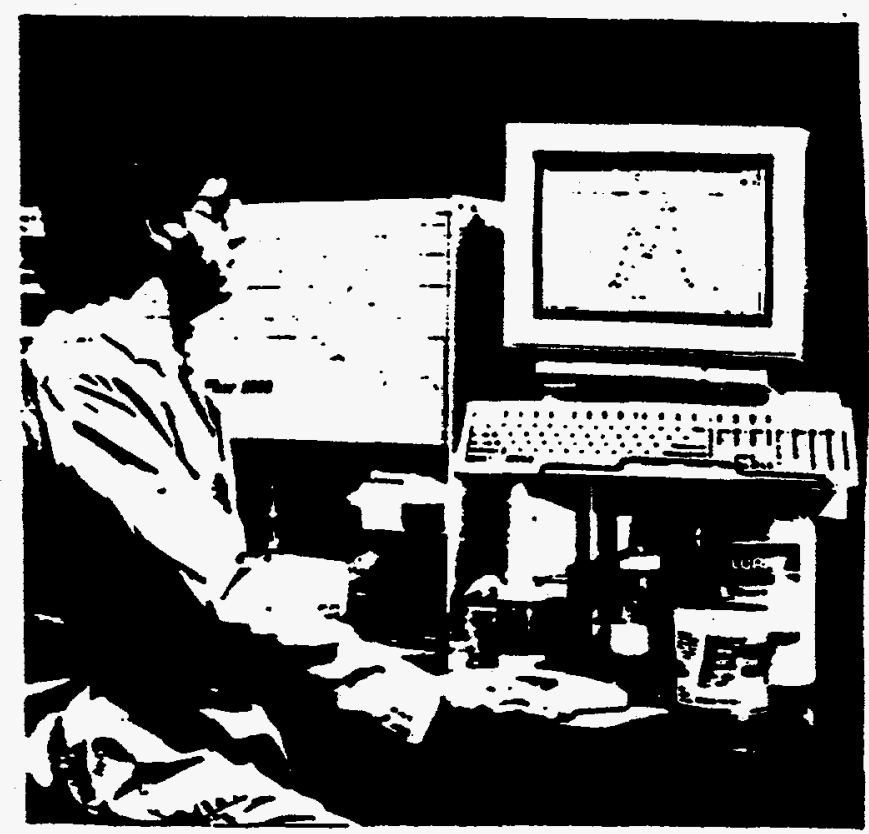

Figure 2 Photograph of AcoustoPhor ${ }^{\text {TM }}$ 8000 showing sample being installed and PSD displayed on screen.

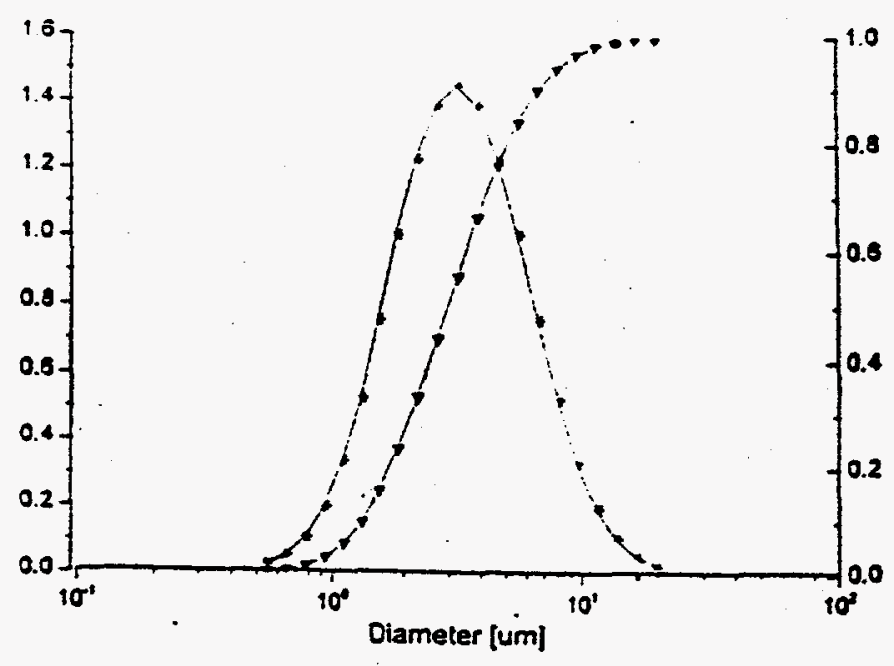

Figure 4 Particle size distribution for 50 wt $\%$ Portland cement slurry Figure 3 Attenuation Spectra of Poth
cement slumies from 10 to 76 wt. \%. 


\section{G UES Tinteruiem}

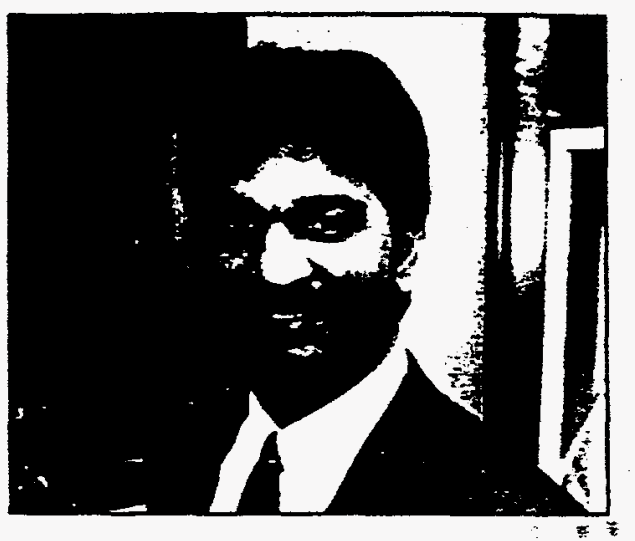

Introduction

Professor Hemant Pendse was the Principal Investigator in the DOE funded program which resulted in the development of the AcoustoPhor 8000 . His association with Pen Kem began ten years ago with his work on electrical effects associated with sound propagation through colloids.

Hemant received his Ph.D. in Chemical Engineering from Syracuse University. He went on to join the faculty at the University of Maine, where he received the Dow Outstanding Young Engineering Faculty Award (ASEE) in 1982. Dr. Pendse is currently the Director of the Industrial Process Control Sensor Systems (IPCOSS) program at the university, and is active in the colloid science community through TAPPI, Fine Particle Society, 1\&EC Division of ACS and AlChE Particle Technology Forum.
Interview: March 26, 1993

Pen Kem: What new advances are necessary either in the under-. standing of colloid science or in new instrumentation techniques?

Prof. Pendse: Characterization of concentrated colloids in terms of charge and size is very important. Having to dilute samples of practical interest and then extrapolate the results to end-use conditions is a major impediment. Instruments which can provide charge, size and shape information simultaneously at realistic particle concentrations can better address the needs of chemical and other processing industries.

Pen Kem: What should be the roles of government and industry in the development of colloid science and associated instrumentation?

Prof. Pendse: Colloid science is an interdisciplinary field which crosses the boundaries of chemistry, chemical engineering, and physics. Instrumentation aspects also bring electrical engineering and software development into the picture. Government and industry could forge linkages among professionals with such diverse backgrounds and experiences.

Du Pont's Particle Science and Technology Center (PARSAT) is a good example of such an association, although their focus is more on powder and slurry technology than colloids. Our own experience shows that the early involvement of people from the manufacturing sector is also important for instrument development. Growing emphasis on matching federal funding with industrial cost sharing, along with collaborative technical interactions at early R\&D stages will certainly help.

We cannot afford to depend solely on the efforts of small companies for the development of novel instrumentation. Such research needs to be fostered on a broader scale, with the results being disseminated through the entire colloid community. A good example of such an effort was the recent Electroacoustics Workshop sponsored by NIST.

Pen Kem: What was the most interesting or rewarding aspect of your collaboration with Pen Kem?

Prof Pendse: I would have to say my sabbatical at Pen Kem during 1987-88. I got to see several scientific instruments in various stages of development and had an opportunity to see many of them used in diverse ways in industrial environments. The synergy created by bringing together creative individuals to develop colloid instrumentation was quite exciting.

\section{AcoustoPhor APT 8000 (continued)}

\section{Typical Data}

Figure $i$ shows particle size distributions for concentrated cement dispersions with and without surfactant. This data shows the change in the degree of flocculation to the addition of the surfactant. To our knowledge, it is the first time such particle size distributions have been made on such a concentrated ( $50-60 \%$ by weight) cement system.

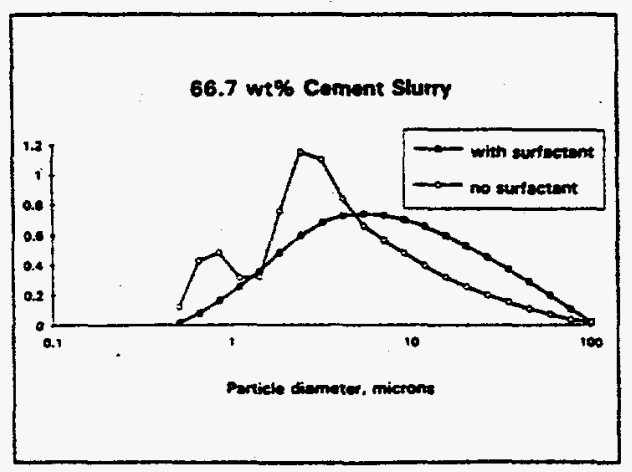

Figure 1: Particle size distribution

\section{Lab version now available}

If you are interested in exploring whether this technique might be suited to your application, contact our Application Lab today. 
PSD Sensor Project including Shape Characterization

\title{
II. Background on Ultrasonic Spectroscopy for PSD Analysis
}

\author{
Arvind Sharma and Hemant P. Pendse, University of Maine, Orono, ME 04469
}

\section{Section Summary}

Using measurements of attenuation coefficients over a wide frequency range one can obtain particle size distribution (PSD) functions for concentrated colloidal suspensions. This paper deals with experimental aspects of ultrasonic spectroscopy. Recent activity in instrumentation for ultrasonic spectroscopy of emulsions and suspensions is briefly reviewed. The measurement issues related to calculations of attenuation coefficients using tone-burst signals are outlined showing significance of using the variable pathlength mode of operation, broadband transducers, and wide dynamic-range signal processing hardware. Attenuation coefficient spectra on titanium dioxide slurries of widely different solids concentrations are analyzed using a theoretical model suitable for high particle volume fractions and a wide range of sound frequencies. A simple equivalent acoustophoretic diameter is suggested to help in interpretation of particle size analysis based on ultrasonic spectroscopy. Sensitivity analysis of attenuation coefficient spectra in relation to changes in the PSDs is presented, outlining a direct approach to obtaining parameters of PSD functions of assumed form using techniques of constrained nonlinear optimization.

\section{Introduction}

One of the early commercial instruments developed for particle size measurement using ultrasound is the PSM-400 system of Armco Autometrics introduced in 1973 for mineral grinding circuits. It employs two transducer pairs, each pair operating at a fixed frequency, and an empirical model to provide an indication of particle size and slurry concentration. Another commercial measurement system, SDE-21, was introduced by ProAssist in 1985. It consists of a signal processor which is connected to the ArmcoAutometrics' electronics and air eliminator. By measuring the attenuation of sound at several frequencies in the range from 1 to $6 \mathrm{MHz}$, the SDE-21 system provides size distribution data for five size fractions. Both PSM-400 and SDE-21 systems have been used to monitor particle size in mining industry, for slurries with mean diameters between 40 to $100 \mu \mathrm{m}$.

In 1990, Parker et al. [1] reported a computer-controlled, variable-gap laboratory apparatus to measure attenuation coefficients of concentrated slurries (at volume fractions up to $40 \%$ ) for frequencies ranging from 5 to $40 \mathrm{MHz}$. They also reported PSD estimates obtained by deconvolution involving Fredholm integral equations. Later, Pen Kem in a joint research project with the University of Maine, developed and tested a prototype of the custom designed sensor electronics and computer system with measurement software capable of programmed ultrasonic spectroscopy. The slurry characterization chambers and the on-line sample delivery system were developed by P\&G Systems and Techna Machine. During this project, one on-line and two laboratory sensor prototypes were 
developed and tested extensively in laboratory and production settings using several industrial mineral pigment slurries. The prototype sensor instrument system was tested successfully in the University of Maine and DuPont research laboratories on several submicron particle suspensions. An on-line prototype was evaluated at a commercial facility for titanium dioxide pigment production during several months of field testing. Field evaluation has shown that the new ultrasound based PSD sensor system is capable of handling several types of mineral pigment slurries commonly encountered in industry under processing concentrations. During the field tests, the system was reported to have operated reliably as an on-line monitoring tool, during protracted runs of continuous operation extending over several days, providing data sensitive to changes in the processing conditions as described by Pendse and Sharma [2].

In 1993, Pen Kem introduced a new commercial laboratory instrument, called AcoustoPhor 8000 Particle Analyzer, for monitoring PSDs of concentrated colloidal slurries without dilution, with particle diameters ranging from 0.01 to $10 \mu \mathrm{m}$. The instrument is based on estimation of PSD functions using measurements of ultrasound attenuation coefficients at several frequencies over 1 to $100 \mathrm{MHz}$. The mathematical algorithms employed in the inversion of measured attenuation coefficient spectra utilize the results of theoretical models developed to predict the behavior of ultrasound as it passes through a slurry of a known concentration, as high as 60 volume percent. Ongoing research work at the University of Maine in this area is documented in three recent dissertations by Strout [3], Han [4], and Sharma [5]. Strout's thesis [3] deals with the development of the viscous coupling coefficient required in the coupled phase continuum framework based on the oscillating sphere-in-cell model. Han [4] modified Strout's work by incorporating effects of particle shapes in terms of the size dependent aspect ratios of equivalent spheroids. Han [4] also developed a unified coupled phase continuum (UCPC) model that accounts for viscous and thermal couplings using frequency and concentration dependent coupling coefficients. The UCPC model is capable of predicting complex wave numbers for both low and high density-contrast solid-fluid suspensions, as well as liquid-liquid emulsions.

\section{Recent Activity in Instrumentation for Ultrasound Spectroscopy}

Hawley [6] designed one of the first laboratory measurement systems suitable for analyzing ultrasonic attenuation spectra of suspensions. It was used in two configurations corresponding to two frequency ranges: a low and intermediate range from 3 to $87 \mathrm{MHz}$, and a high frequency range covering 75 to $165 \mathrm{MHz}$. Operation in both ranges was accomplished for the most part with the same mechanical components in slightly different orientation. Experimental data was collected on attenuation of a suspension of polystyrene latex spheres in a mixture of $\mathrm{H}_{2} \mathrm{O}$ and $\mathrm{D}_{2} \mathrm{O}$. Later, Allegra [7] used the setup to analyze various aqueous emulsions.

A lot more activity in instrument development has been reported during the past decade in the area of ultrasound spectroscopy of suspensions and emulsions. Riebel and Löffler [8] reported on a laboratory apparatus covering the frequency range from 0.5 to $90 \mathrm{MHz}$, 
which they used to analyze particles ranging from about 20 to $1000 \mu \mathrm{m}$ in diameter, with particle concentrations up to $10 \%$ by volume. To illustrate the performance of ultrasonic spectrometry, they have presented some measurements with glass beads. The ultrasonic extinction was measured up to $50 \mathrm{~dB}$, depending on the frequency used. This work forms the basis for a commercial instrument, called OPUS, introduced by Sympatec GmbH. Kräuter and Riebel [9] reported on the use of ultrasonic spectrometry as an in-line method of particle size analysis. Their system evaluates ultrasonic extinction as a function of ultrasonic frequency in the range from 1 to $100 \mathrm{MHz}$ using variable pathlength ranging from 1 to $12 \mathrm{~mm}$. Schaafsma [10] used acoustic attenuation spectroscopy to analyze concentration and size of suspended sediment particles, i.e., silt, clay, and sand, with average sizes between 5 and $250 \mu \mathrm{m}$. The attenuation spectra cover the frequency range from about 1 to $100 \mathrm{MHz}$ using six broadband transducer pairs to span the total frequency range. Alba et al. [11] have reported on characterization of oil/water emulsions using their UltraSpec Particle Size Analyzer. The range of the concentrations of the emulsions analyzed is from $0.5 \%$ to $10 \%$ by volume, with the particle diameters in the range of 0.01 to $1000 \mu \mathrm{m}$. The patent for the UltraSpec particle size analyzer states a frequency band of 1 to $200 \mathrm{MHz}$. Boxman et al. [12] report on a flow-through cell which can be used for analyzing commercial slurries. The frequency range of measurement is said to be from 2 to $70 \mathrm{MHz}$. For the case of concentrated slurries, the upper frequency limit is said to drop to about $50 \mathrm{MHz}$ due to the signal-to-noise limitations.

\section{Experimental Details}

Focusing our attention on aqueous slurries of inorganic pigments with high densitycontrast, we studied well-dispersed aqueous suspensions of titanium dioxide and kaolin clay pigments. The titanium dioxide test slurries were prepared by careful dilution of a pre-dispersed R-940 slurry formulated at $75 \mathrm{wt} \%$ solids. Independent PSD analyses of the test slurries using SediGraph 5100 confirmed that all of titanium dioxide test slurries are characterized by a single particle distribution curve independent of their particle concentrations. The titanium dioxide SediGraph PSD corresponds to median diameter of $0.35 \mu \mathrm{m}$, modal diameter of $0.31 \mu \mathrm{m}$, and geometric standard deviation (gsd) of 1.6.

Ultrasonic characterization was performed using Techna Machine's variable pathlength, flow-through, slurry characterization chamber (TM-SCC) and an early prototype of Pen Kem's ultrasonic spectrometer measurement system (PK8000). Total attenuation, termed 'sensor loss', was measured for 21 gaps at 18 frequencies using tone-burst signals with a test slurry circulating though the chamber. Using the sensor loss tables, attenuation coefficient spectra were calculated for the test slurries of different particle concentrations. This procedure is illustrated in Figures 1(a) and 1(b), where the total attenuation (in dB) is plotted against the gap (i.e., pathlength in $\mathrm{mm}$ ) for different operating frequencies for the most concentrated titanium dioxide test slurry. Details of the procedure are given by Sharma [5] in his dissertation. Useful sensor loss measurements can be as high as 150 $\mathrm{dB}$, limited only by the signal-to-noise ratio. Only a small fraction of the raw data in the total sensor loss table is useful for calculations of the attenuation coefficients, with the useful gap-ranges varying with the operating frequency. Attenuation coefficients 
correspond to the slopes of the linear sub-regions shown in Figure 1(b). The zero-gap sensor losses of the particular transducer pair used in the chamber are about $40 \mathrm{~dB}$ at the resonant frequency of the transducers, increasing to about $100 \mathrm{~dB}$ at 1 or $100 \mathrm{MHz}$. For low frequencies, sensor loss data is useful for gaps ranging from 5 to $20 \mathrm{~mm}$, yielding 8 data points for the linear regression analysis. For the last three high frequencies, however, the useful data for this chamber appears to be limited to the two smallest gaps. For chambers with a much smaller minimum gap, of about $0.1 \mathrm{~mm}$, one obtains many more useful data points, with gaps ranging from 0.1 to $0.6 \mathrm{~mm}$, at very high frequencies; even with large sensor losses corresponding to the zero-gap limit. A wide-band operation is possible over the frequency range of 1 to $100 \mathrm{MHz}$ for a single attenuation coefficient spectrum that covers a range from 10 to $1000 \mathrm{~dB} / \mathrm{cm}$, when appropriate variable gap set up is used in combination with sensor electronics capable of measuring losses as high as $150 \mathrm{~dB}$. The measured attenuation spectrum corresponding the data shown in Figure 1, for the $75 \mathrm{wt} \%$ test slurry, is shown in Figure 2 in a normalized form along with some additional information.

After the first measurement on a new test slurry, subsequent measurements can be done faster by dropping the unused frequency-gap combinations resulting in significant time savings and increased speed of operation. In our on-line system, we used two slurry characterization chambers with transducer pairs corresponding two different resonant frequencies. Attenuation spectra obtained from the sensor loss data obtained from two chambers can be combined to yield a more robust measurement, while providing redundancy in the measurement system. The coefficients of variation (standard deviation/mean), for the slopes obtained from six repeat measurements on a given test slurry are found to be less than $2 \%$. Attenuation coefficient spectra with such low coefficients of variation, covering a wide range of frequencies, can be used to obtain particle size information. Similar measurements on kaolin clay slurries are discussed by Han [4] in his dissertation. Detailed analysis of attenuation coefficient spectra for kaolin slurries, in terms of size-dependent shape factor distribution, was provided by Han and Pendse [13].

\section{IIlustrative Results}

Here we focus on the titanium dioxide slurry data since it covers particle concentrations ranging from 13 to $75 \mathrm{wt} \%$. The theoretical predictions shown here are based on the coupled phase continuum framework, using the oscillating sphere-in-cell model developed by Strout [3] for calculating the viscous coupling coefficient. The predictions are obtained using an assumed PSD specified by the two parameters of a log-normal function, namely geometric mean ( $\mathrm{gm}$ ) and geometric standard deviation ( $\mathrm{gsd}$ ). The log-normal PSD function is given as:

$$
\begin{aligned}
& p(x)=\frac{1}{\sqrt{2 \pi} \ln g s d} \exp \left[-\frac{1}{2} \cdot\left(\frac{x-\ln g m}{\ln g s d}\right)^{2}\right] \\
& \ldots \text { where } \quad x=\ln d
\end{aligned}
$$


Here, $p(x) d x$ is the fractional mass of particles with sizes corresponding to the subinterval $x$ to $(x+d x)$, and $d$ denotes the particle diameter. Corresponding PSD on number basis is also of the log-normal form with identical geometric standard deviation, gsd, but a different geometric mean, $g m_{\text {number }}$, which is related to $g m$ as:

$$
\ln \left(g m_{\text {number }}\right)=\ln (g m)-3 \cdot[\ln (g s d)]^{2}
$$

Based on the analysis of the frequencies corresponding to the peaks for the normalized attenuation coefficient spectra, a convenient equivalent acoustophoretic diameter, ead, can be identified with the two parameters, gm and gsd, of a log-normal PSD on mass basis, as suggested by Sharma [5]:

$$
\ln (e a d)=\ln (g m)-\frac{3}{8} \cdot[\ln (g s d)]^{2}
$$

The ead is analogous to the so called 'surface-volume' mean diameter, for which the coefficient in the ln-squared term is $-1 / 2$ instead of $-3 / 8$.

\section{Discussion}

Figure 2 shows the frequency response of attenuation coefficients for two test slurries.

The attenuation coefficients have been divided by the corresponding volume fractions $(\phi)$ and frequencies $(f)$, for convenience. In this form, the peak in the normalized spectrum at about $15 \mathrm{MHz}$ for $13 \mathrm{wt} \%$ slurry is apparent. The peak frequency for the normalized spectrum for $75 \mathrm{wt} \%$ slurry appears to be above $100 \mathrm{MHz}$. The two spectra corresponding to 13 and $75 \mathrm{wt} \%$ solids concentrations (i.e., 3.5 and $42.3 \mathrm{vol} \%$ ) are quite different in magnitude and shape, even when normalized by the corresponding particle volume fractions, emphasizing the nonlinear volume fraction effects. The need to properly account for dependence of ultrasonic attenuation spectra on particle volume fraction is obvious. Also shown in Figure 2 are theoretical predictions based on three test PSDs. These predictions illustrate the effects of a shift in gm at fixed gsd of 1.65 , and also a shift in $g s d$ at a fixed $g m$ of $0.33 \mu \mathrm{m}$. Upward shift in the peak frequency from 8 $\mathrm{MHz}$ to $15 \mathrm{MHz}$ with the downward shift in the $g m$ diameter from 0.33 to $0.27 \mu \mathrm{m}$ is especially clear from the model predictions for the $13 \mathrm{wt} \%$ slurry. Broadening of the PSD is reflected in the broadening of the attenuation spectra for both cases.

-Sensitivity of attenuation coefficient spectra to the changes in the PSD is studied in detail by Sharma [5]. Calculations were made to study sensitivity to changes in the gm and the gsd parameters of the assumed PSDs. It may be noted that the four sets of parameters chosen have $10 \%$ deviations in $g m$ and $g s d$ from the central base case $(g m=1.30 \mu \mathrm{m}$ and $g s d=1.5$ ). The five test PSDs cover the ead range of 0.245 to $0.319 \mu \mathrm{m}$. Quantitative results on the sensitivity analysis are depicted in Figure 3, where the composite rootmean-squared relative differences over all concentrations are plotted as a function of frequency. Figure 3 shows that the two test PSDs with a low gsd of 1.35 ( $\square$ and $\diamond$ ) result in high relative deviations where as the test PSDs with high a gsd of 1.65 ( $\triangle$ and $\nabla$ ) yield a better agreement between the predicted and measured spectra.

For a given test slurry, one can fine-tune $g m$ and $g s d$ values using optimization techniques developed for parameter search in a multi-dimensional space, with a suitable objective function involving appropriate weighted average magnitude of the relative 
deviations corresponding to different frequencies. One can also include measures related to peak location mis-match in the objective function definition. Rather than using a unimodal log-normal PSD, one can optionally use any multi-modal PSD function and obtain corresponding PSD parameters along with the relative contributions of individual mode. The parameter estimation problem is formulated as a constrained nonlinear optimization problem in terms of a scalar objective function which depends on the parameters of a PSD function. Use of direct optimization techniques for obtaining best estimates of parameters for an assumed PSD functional form may be more robust compared with solving an integral equation without any assumptions about the functional form of the PSD. PSD estimation results based on such deconvolution of the governing integral equation were discussed previously by Parker et al. [1], and Pendse, Strout and Sharma [14].

The extent of sound attenuation depends on the length of the acoustic path, the frequency of sound, the concentration and sizes of the particles, and the physical properties of the particulate and fluid phases. Computer-controlled, programmed ultrasonic spectrometers that use flow-through slurry characterization chambers with variable pathlengths allow one to rapidly measure attenuation coefficient and sound speed spectra of concentrated suspensions over a wide range of frequencies. Availability of a predictive, theoretical model that accounts for hydrodynamic interactions underlying the acoustophoresis in suspensions with moderate to high particle volume fractions, over a wide range of frequencies, has made it possible to interpret measured attenuation coefficient spectra in terms of PSDs. Sensitivity analysis of attenuation spectra in response to changes in the PSD parameters shows that one can use optimization techniques for parameter estimation in terms of a constrained nonlinear optimization problem.

\section{References}

1. D. Parker, R. M. Lec, H. P. Pendse, and J. F. Vetelino, IEEE Ultrasonics Symposium (1990) 11-14.

2. H. P. Pendse and A. Sharma, Part. Part. Syst. Charact., 10 (1993) 229-233.

3. T. A. Strout, Ph. D. Thesis, University of Maine, Orono (1991).

4. W. Han, Ph. D. Thesis, University of Maine, Orono (1995).

5. A. Sharma, Ph. D. Thesis, University of Maine, Orono (1995).

6. S. A. Hawley, Ph. D. Thesis, University of Illinois, Urbana (1967).

7. J. R. Allegra, Ph. D. Thesis, Harvard University, Cambridge (1970).

8. U. Riebel and F. Löffler, Part. Part. Syst. Charact., 6 (1989) 135-143.

9. U. Kräuter and U. Riebel, Proc. of the First International Particle Technology Forum, American Institute of Chemical Engineers, Denver, 17-19 August 1994, Part I, 30-35.

10. A. S. Schaafsma, Proc. of Ultrasonics International, Madrid (1989).

11. F. Alba, C. L. Dobbs and R. G. Sparks, Proc. of the First International Particle Technology Forum, American Institute of Chemical Engineers, Denver, 17-19 August 1994, Part I, 36-46. 
PSD Sensor Project including Shape Characterization

12. A. Boxman, D. M. Scott and C. E. Jochen, Proc. of POWTECH 95, Nürnberg (1995) 37-46.

13. W. Han and H. P. Pendse, Proc. of the First International Particle Technology Forum, American Institute of Chemical Engineers, Denver, 17-19 August 1994, Part I, 142-147.

14. H. P. Pendse, T. A. Strout and A. Sharma, in S. G. Malghan (Ed.), Electroacoustics for Characterization of Particulates and Suspensions, NIST Special Publication, 856 (1993) 23-39. 
PSD Sensor Project including Shape Characterization
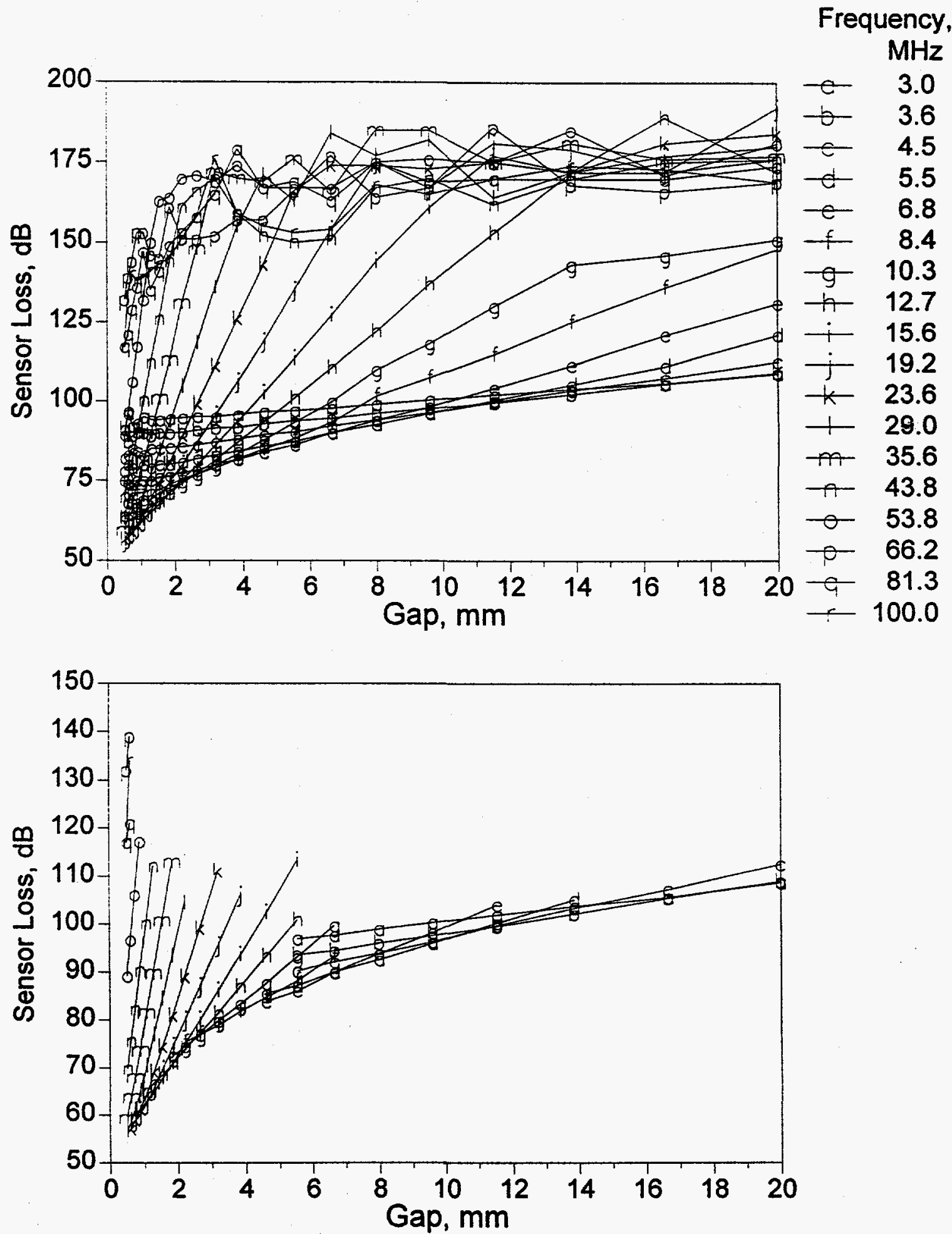

Figure 9 Raw data on sensor loss as a function of gap, used in calculation of attenuation coefficients for $75 w t \%$ titanium dioxide test slurry: (a) Total data (b) Subset used for slope calculations. 
PSD Sensor Project including Shape Characterization

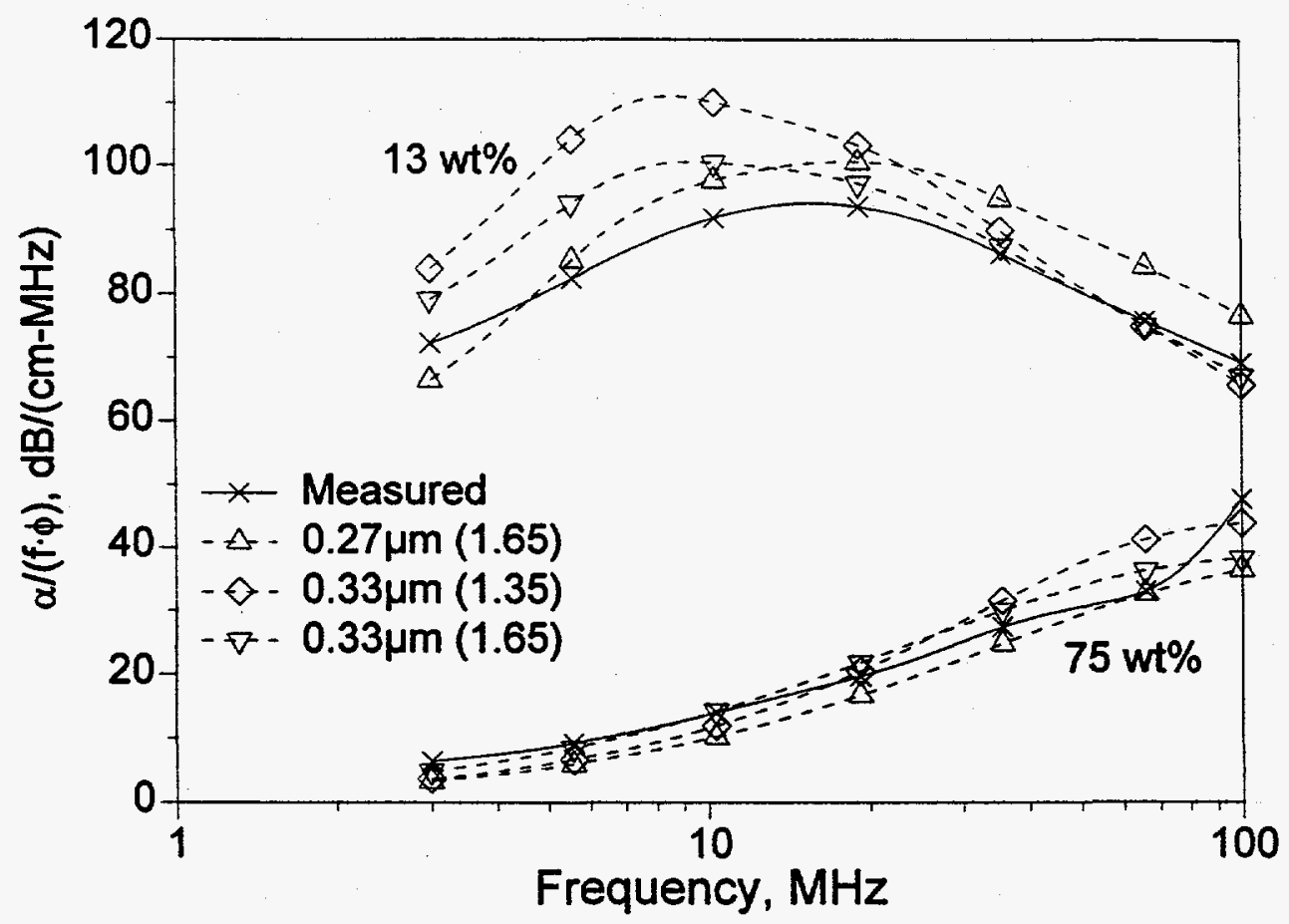

Figure 10 Attenuation coefficient spectra for two test slurries compared to predictions based on three assumed test PSDs. 
PSD Sensor Project including Shape Characterization

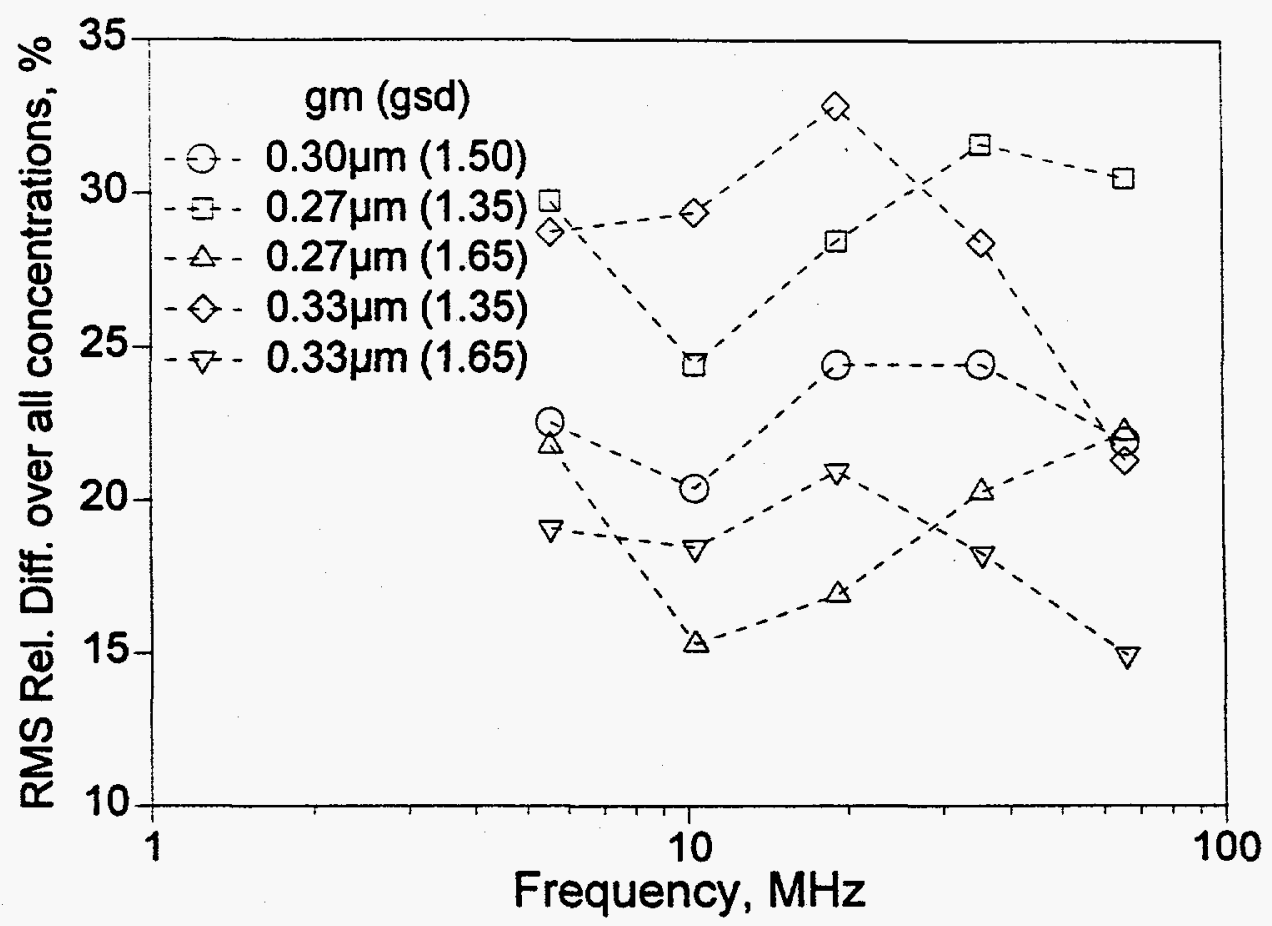

Figure 11 Sensitivity analysis results for root-mean-squared relative differences between predicted and measured attenuation coefficients over all concentrations for five assumed test PSDs. 


\section{Ultrasonic Spectroscopy for Shape Analysis}

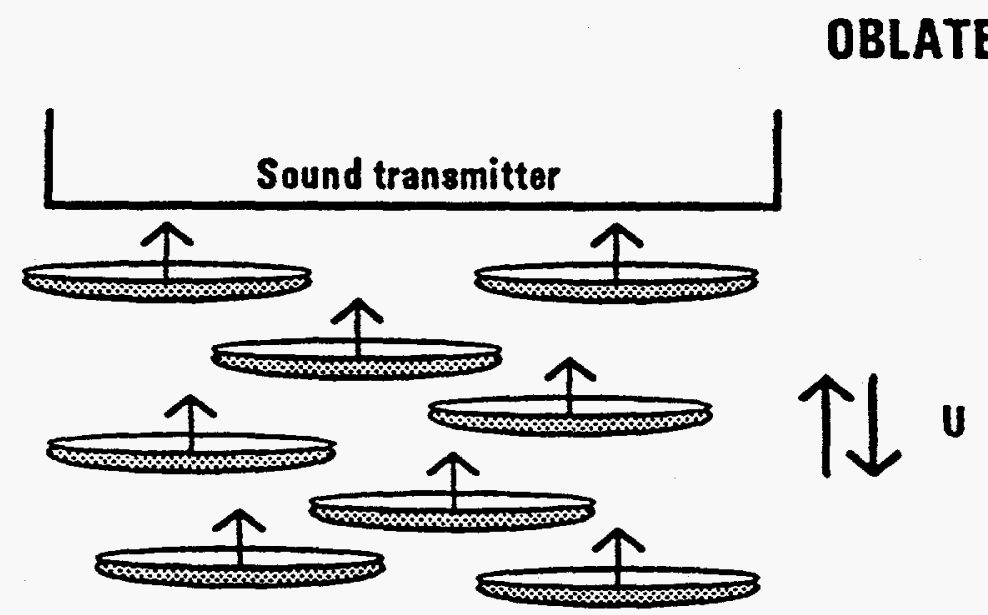

Sound receiver

Broadside to sound Field

(Parallel Orientation)

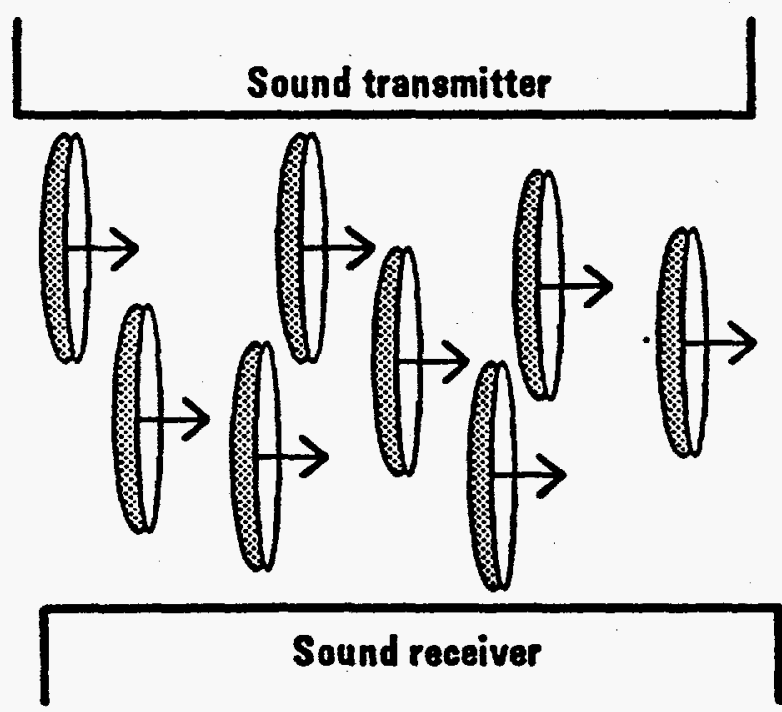

Edgewise to sound field

(Perpendicular Orientation) 


\section{Analysis of Shape Effects: Tests using Clay Slurries}

Wei Han and Hemant P. Pendse, University of Maine, Orono, ME 04469

\section{Section Summary}

Results of laboratory investigation are presented here on the frequency-dependent ultrasound attenuation coefficient of suspensions containing spheroid particles of high density contrast, with particular attention to the effects of particle size and shape distributions (PSSD). The theoretical analysis is based on the coupled-phase framework that takes into account the effects of particle size and shape through the viscous coupling coefficient. It is shown that predictions based on realistic PSSDs allow one to interpret measured ultrasonic spectra for kaolin-water slurries.

\section{Introduction}

Clay pigment particles in general have a platelet shape. For platelet or disk-like particles the shape factor can be defined as the thickness to face diameter ratio, also called the aspect ratio, $R$.. We obtained attenuation coefficient spectra over a wide frequency range using slurries of different particle concentrations. These slurries were prepared by careful dilution of a pre-dispersed kaolin slurry formulated at $35 \mathrm{wt}$ \% solids.

The present model $[1,2]$ uses the coupled-phase framework with the viscous coupling coefficient related to the drag force experienced by an oscillating sphere in cell. In an earlier study $[3,4]$ this model was tested using well-dispersed titanium dioxide suspensions. This model has now been modified to take into account the effects of nonsphericity of suspended particles. This allows us to quantitatively account for a particle size and shape distribution (PSSD) appropriate for kaolin clay.

\section{Results and Analysis}

Here we present illustrative analysis of the ultrasonic spectra of clay suspensions. Measured ultrasound attenuation spectra of concentrated clay suspensions are interpreted in terms of suitable size and shape distributions. Figure 12 shows attenuation coefficient data for three concentrations $(2.9,7.61$ and 16.9 vol. \% solids) as functions of sound frequency, and similar data for two operating frequencies $(6.85$ and $35.7 \mathrm{MHz})$ as functions of particle concentration. The excess attenuation coefficients range from 2 to $200 \mathrm{~dB} / \mathrm{cm}$ increasing with increasing frequency and particle concentration.

Figure 12 also shows model predictions, which are based on test PSSDs shown in Figure 13. A given sedimentation-based particle size distribution (PSD) corresponds to five different PSDs when expressed in terms of volume equivalent sphere diameters corresponding to five test shape factor distributions shown in Figure 13. Random orientation was assumed to apply to sedimentation as well as acoustophoresis. The test 
distribution PSSD1, with constant aspect ratio R of 1, corresponds to the assumption of spherical shape where the volume equivalent diameters, $\mathrm{D}$ and the sedimentation equivalent diameters, $D_{\text {sed }}$ become identical. The test distributions PSSD2 and PSSD3 assume constant $R$ of 0.05 and 0.10 , respectively. The assumption of size-independent aspect ratio is obviously an over simplification for a natural pigment like kaolin. Very small kaolin particles can be fragments with shape factors in the range of 0.2 to 1 . The coarse kaolin particles can be 'booklets' or aggregates with shape factors in the range of 0.1 to 0.4 . In the intermediate size range, one encounters natural platy particles of shape factors ranging from 0.1 to 0.01 . Based on these considerations, we have constructed two test distributions PSSD4 and PSSD5 with size dependent shape factors corresponding to basic plate thickness of $70 \mathrm{~nm}$ and $140 \mathrm{~nm}$, respectively.

Predictions based on the assumption of spherical shape are in agreement with the experimental data for dilute slurries at low frequencies. It is clear that effects of nonsphericity become more prominent as the sound frequencies (or particle concentrations) increase. The overall agreement between theoretical predictions and experimental measurements is remarkably well when shape factors of non-spherical particles are taken into account. In particular, predictions corresponding to PSSD4 appear to match experimental data over wide ranges of frequency and particle concentration. This shape distribution corresponds to $70 \mathrm{~nm}$ thick platelets. The preliminary analysis indicates that attenuation spectra for clay slurries with sufficiently high solids volume fractions can allow one to discriminate among several realistic shape factor distributions, especially in the high frequency region. Complete details have been documented in the Chapter 5 of Han's dissertation []]

\section{References}

1. Pendse, H.P. and T.A. Strout, Proc. Sensor Expo West, 205B (1990).

2. Strout, T.A., "Attenuation of Sound in High-Concentration Suspensions," Ph.D. Dissertation, University of Maine, Orono, ME (1991).

3. Pendse, H.P., T.A. Strout and A. Sharma, "Theoretical Considerations in Acoustophoresis Analysis of Concentrated Colloids," in NIST Special Publication No. 856, S.G. Malghan (Ed.) 23 (1993).

4. Sharma A., , "Ultrasonic Spèctroscopy of Solid Liquid Suspensions," Ph.D. Dissertation, University of Maine, Orono, ME (1995).

5. Han, W., "Effects of Particle Shape on Viscous Attenuation in Concentrated Clay Suspensions" in "Viscothermal Coupling Effects on Sound Attenuation in Concentrated Colloidal Dispersions" Ph. D. Dissertation, University of Maine, Orono, ME (1995) 
PSD Sensor Project including Shape Characterization
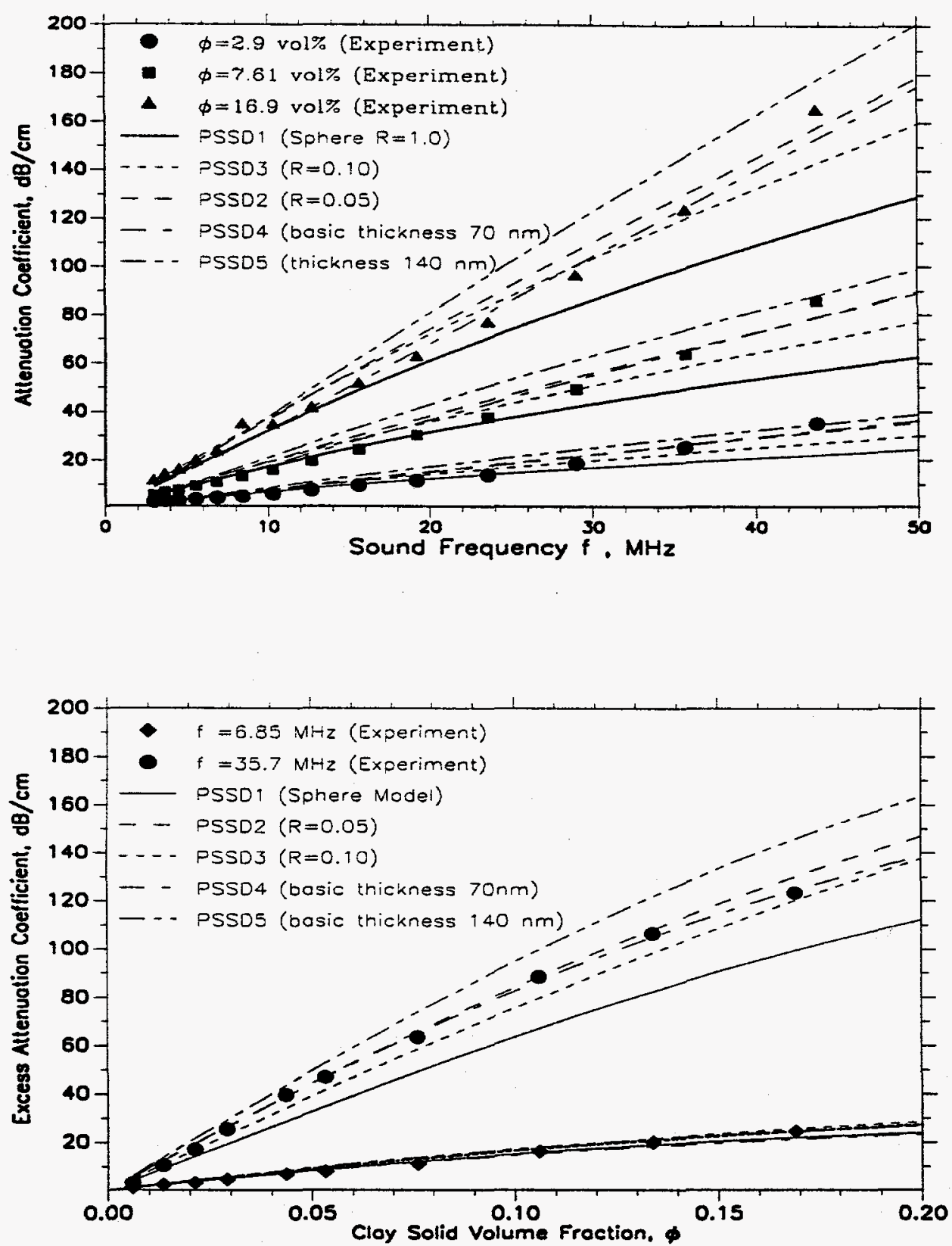

Figure 12 Attenuation coefficient as a function of (a) sound frequency and (b) particle concentration: Comparison of predictions and the experimental data, for five test PSSDs. 
PSD Sensor Project including Shape Characterization
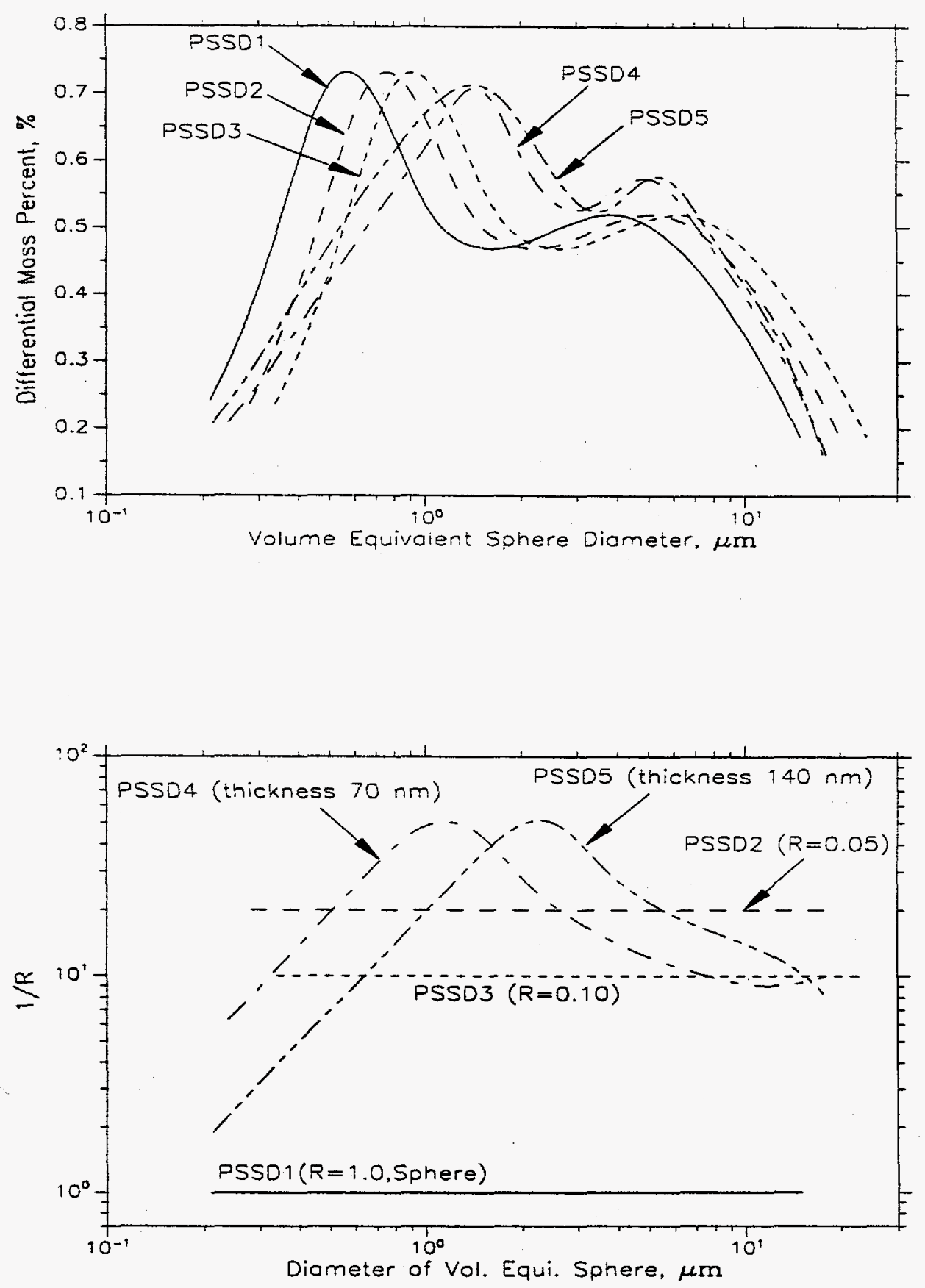

Figure 13 Test particle size and shape distributions (PSSDs): (a) differential size distributions and corresponding size dependent shape factor distributions 


\section{Particle Orientation Evolution in Suspensions subjected to Squeezing Flow}

Timothy C. Bliss and Hemant P. Pendse, University of Maine, Orono, ME 04469

\section{Section Summary}

The orientation evolution of particles in suspension subjected to squeezing flows is described using the Transversely Isotropic Fluid (TIF) model. Analytical solutions for the TIF model are in agreement with classical solutions for the Jeffrey's orbits for spheres in a simple shear flow. For the squeezing flows, the governing differential equations for the TIF model are transformed into finite difference equations using the upwind difference scheme. The unit vector field evolution is quantified in terms of the angle made by the particle's axis of revolution with respect to the approaching piston's face. Effects of the particle's aspect ratio and initial orientation angle distribution on the orientation angle evolution are investigated. In general, it was found that the particles at the off-center locations would approach close to parallel orientations, which they would hold for a short duration before undergoing repeated rotations. Highly non-spherical particles (aspect ratio, $\mathrm{A}>10$ or $\mathrm{A}<0.1$ ) tend to spend considerable time in parallel orientation, but nearly-spherical particles $(0.1<\mathrm{A}<10)$ tend to spend considerable time undergoing repeated rotations of decreasing periods. The line-of-sight averages of the orientation angles provides quantitative measure of the orientation evolution. These can be useful in sensor applications that use squeezing flows, where the sensor response is dependent on particle orientations along the line-of-sight.

\section{Background}

Figure 14 shows the fixed reference frame and a particle orientation vector describing how a particles orientation is related to this fixed coordinate system. Schematics of the sensing zone is shown in Figure 15 for reference. For the purposes of the current work, the only angle of consequence is that describing a particle's rotation from the radial axis, $\beta$. Because axial symmetry is assumed, only a single two-dimensional plane is considered. Every one of the infinite number of two-dimensional planes will have the exact same particle orientation field. The particle center, $\mathrm{C}$, is the origin of the particle orientation vector. The angle between the particles axis of rotation, $\mathrm{Z}^{\prime}$, and its projection on a plane parallel to the midplane, $\overrightarrow{C E}$, is $\beta$. Time-dependent particle orientation field differs considerably from the fluid velocity field, illustrated in Figure 16. The following Table defines orientation terminology.

\begin{tabular}{|l|l|l|l|}
\hline Aspect Ratio, $\mathrm{A}$ & Prolate, $\mathrm{A}>1$ & Oblate, $\mathrm{A}<1$ & Sphere, $\mathrm{A}=1$ \\
\hline $\begin{array}{l}\text { Parallel } \\
\text { orientation }\end{array}$ & $\begin{array}{l}\text { The axis of revolution } \\
\text { is parallel to the } \\
\text { piston face }\end{array}$ & $\begin{array}{l}\text { The axis of } \\
\text { revolution is } \\
\text { perpendicular to the }\end{array}$ & $\begin{array}{l}\text { Parallel orientation } \\
\text { is not defined } \\
\beta=\text { any angle }\end{array}$ \\
\hline
\end{tabular}


PSD Sensor Project including Shape Characterization

\begin{tabular}{|l|l|l|l|}
\hline & $\beta=0^{\circ}$ or $\beta=180^{\circ}$ & $\begin{array}{l}\text { piston face } \\
\beta=90^{\circ} \text { or } \beta=270^{\circ}\end{array}$ & \\
\hline $\begin{array}{l}\text { Perpendicular } \\
\text { orientation }\end{array}$ & $\begin{array}{l}\text { The axis of revolution } \\
\text { is perpendicular to } \\
\text { the piston face } \\
\beta=90^{\circ} \text { or } \beta=270^{\circ}\end{array}$ & $\begin{array}{l}\text { The axis of } \\
\text { revolution is parallel } \\
\text { to the piston face } \\
\beta=0^{\circ} \text { or } \beta=180^{\circ}\end{array}$ & $\begin{array}{l}\text { Perpendicular } \\
\text { orientation is not } \\
\text { defined } \\
\beta=\text { any angle }\end{array}$ \\
\hline
\end{tabular}

\section{IIlustrative Results for oblate and prolate orientation evolution}

Figure 17 shows results for four different particles all located at the same point in a system $\left(R^{*}=0.85, \bar{Z}=0.75\right)$ with an initial gap of $H_{0} / R_{0}=0.25$. The particle with an aspect ratio of 20 rotates quicker at first than a particle with an aspect ration of 0.05 (i.e. 1/20). Parallel orientation of the particle with an aspect ratio of 20 corresponds to $180^{\circ}$. Therefore, it begins much farther from parallel orientation than the particle with an aspect ratio of 0.05 , which is oriented parallel at $90^{\circ}$. As these particles approach parallel orientation, their rates of rotation become very similar.

There is much less difference between oblate and prolate particles as their aspect ratios approach 1.0. Figure 17 shows particles with aspect ratios of 2.0 and 0.5 (i. e. 1/2). Their rotation, although not exactly the same, has a similar form. The particle with an aspect ratio of 2.0 rotates quickly at first. Near an angle of $180^{\circ}$, its rotation slows and then begins to speed up again as it travels away from $180^{\circ}$. For the particle with an aspect ratio of 0.5 , the rotation is slow at first, as it is near an angle of $90^{\circ}$ (parallel orientation). As the particle moves away from $90^{\circ}$, its rotation speeds up. After a time of approximately 0.8 , they rotate in an almost identical manner. It is clear that the particles at the off-center locations approach close to parallel orientations, which they would hold for a short duration before undergoing repeated rotations. Highly non-spherical particles (aspect ratio, $\mathrm{A}>10$ or $\mathrm{A}<0.1$ ) tend to spend considerable time in parallel orientation, but nearly-spherical particles $(0.1<A<10)$ tend to spend considerable time undergoing repeated rotations of decreasing periods.

\section{Conclusions}

It should be noted that the attenuation coefficients of suspensions of spheroids are strongly influenced by particle orientations, as shown by Han. Figures 5.20 and 5.21 from Han's dissertation are reproduced here for easy reference, as Figures 18 and 19. Han denotes the aspect ratio by $R$. The ratio of excess attenuation coefficients for random versus parallel orientation is shown as functions of frequency, $f$, or equivalently $\left(k R_{\text {ves }}=2 \pi . f . R_{\text {ves }} / c_{0}\right) . R_{\text {ves }}$ denotes average volume equivalent radius of the particles. It is clear that the frequency dependence of the attenuation ratio is sensitive to size dependent shape distributions. Information on orientation field evolution associated with squeezing flows can be used to infer shape factors from attenuation spectra obtained in the continuously-changing-gap mode, as compared to those in the multiple-gap-sequence mode. 


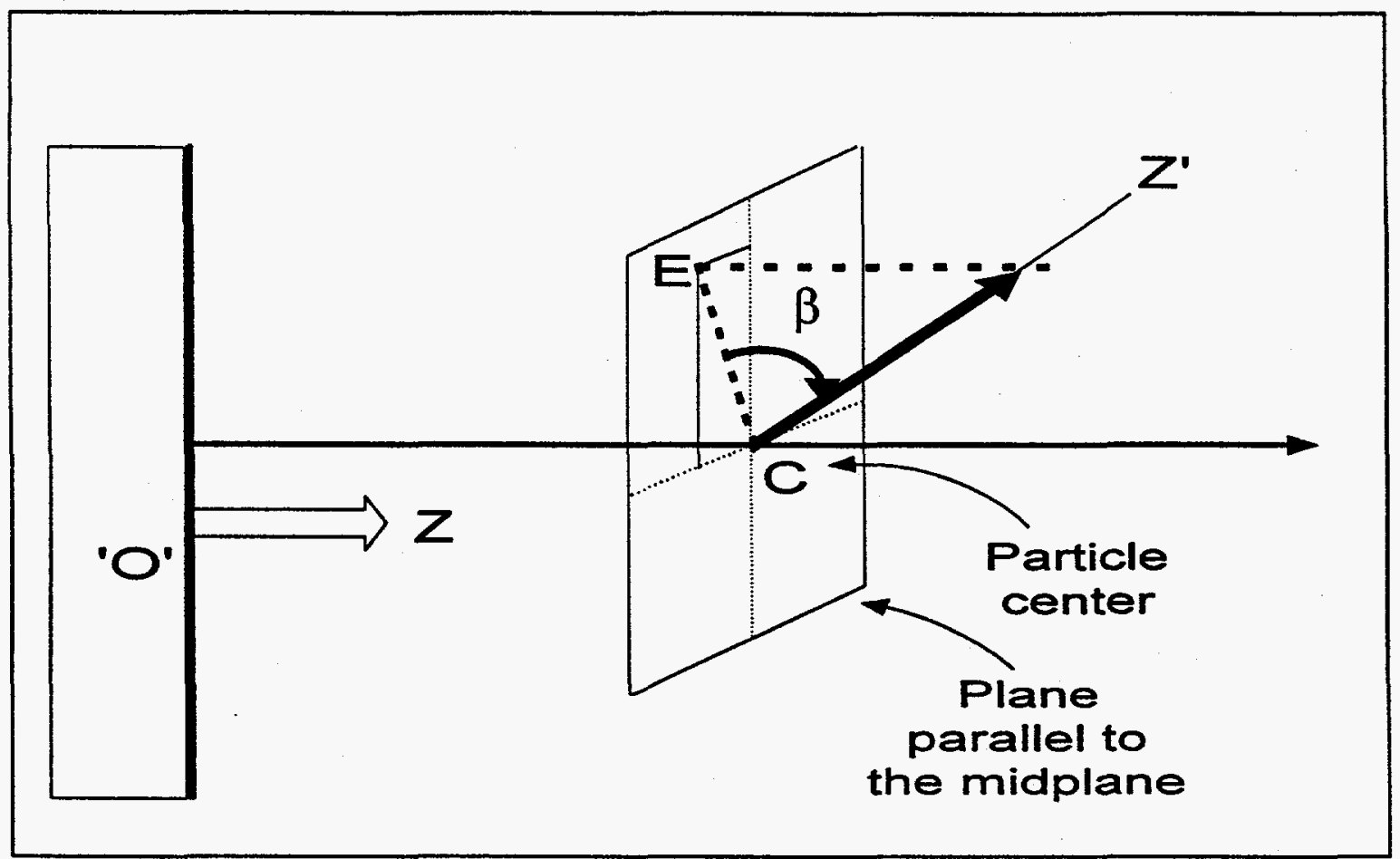

Figure 14 Particle orientation angle, $\beta$ where $\mathrm{CZ}$ ' is the particle's axis of revolution.

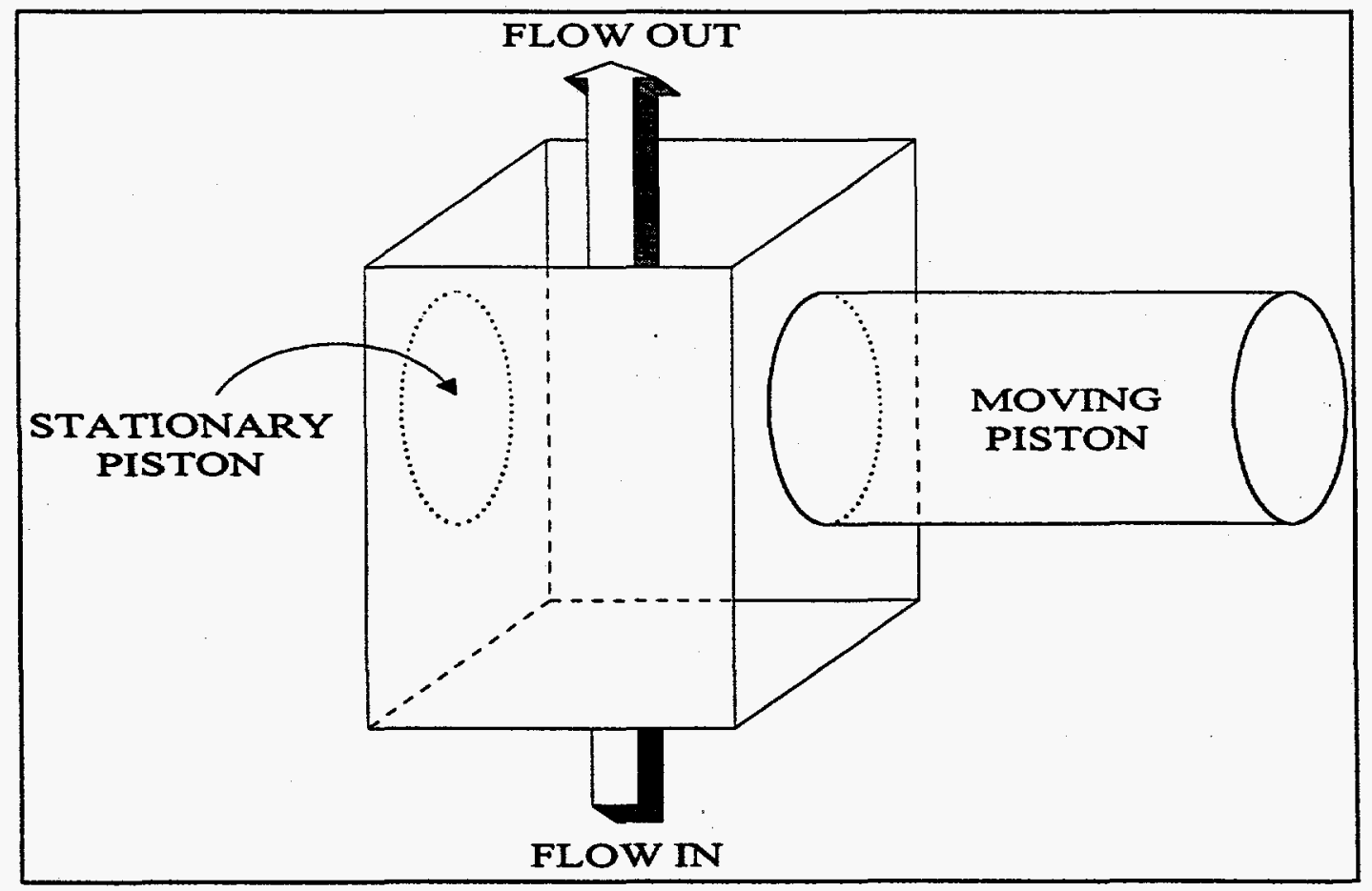

Figure 15 Simple schematic of sensing zone where squeezing flow would be encountered in a continuously decreasing gap mode. 
PSD Sensor Project including Shape Characterization

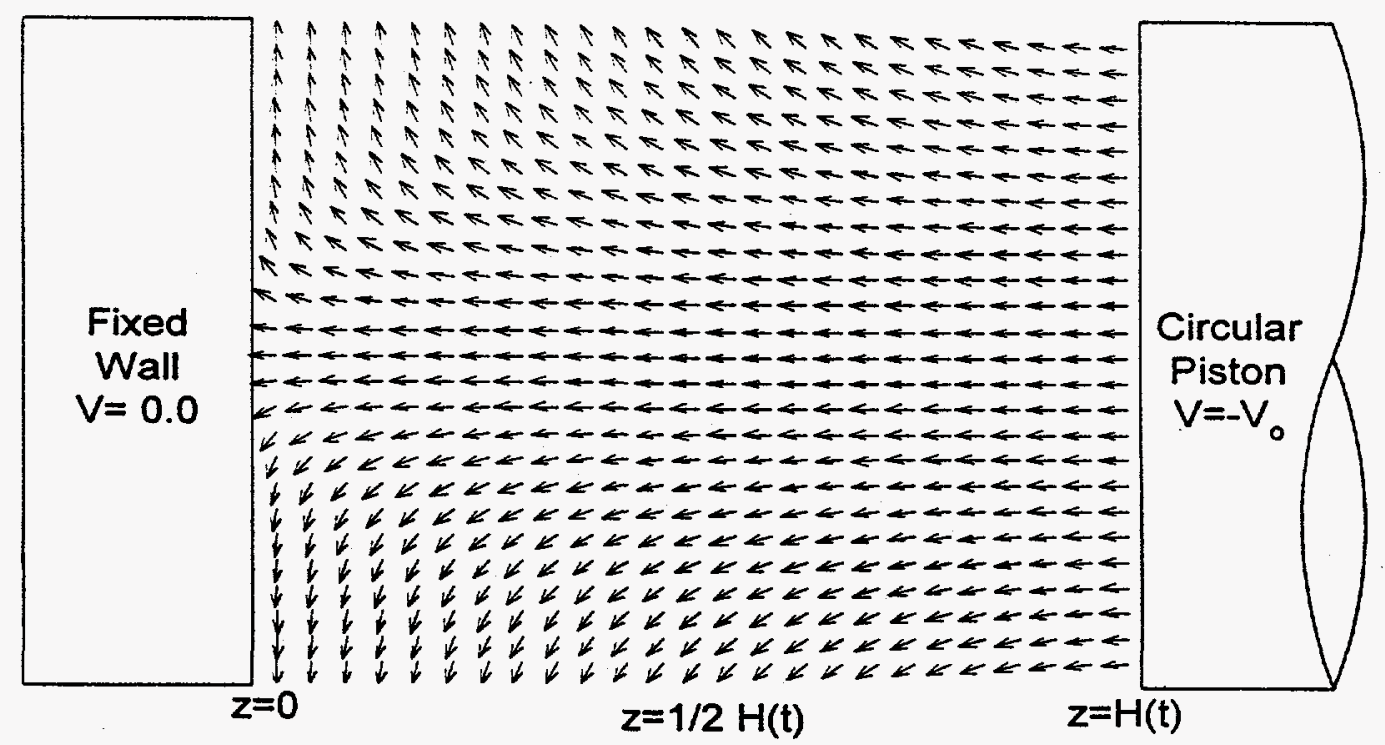

Figure 16 Velocity vector orientation profile between moving piston and wall (shown using unit vectors)

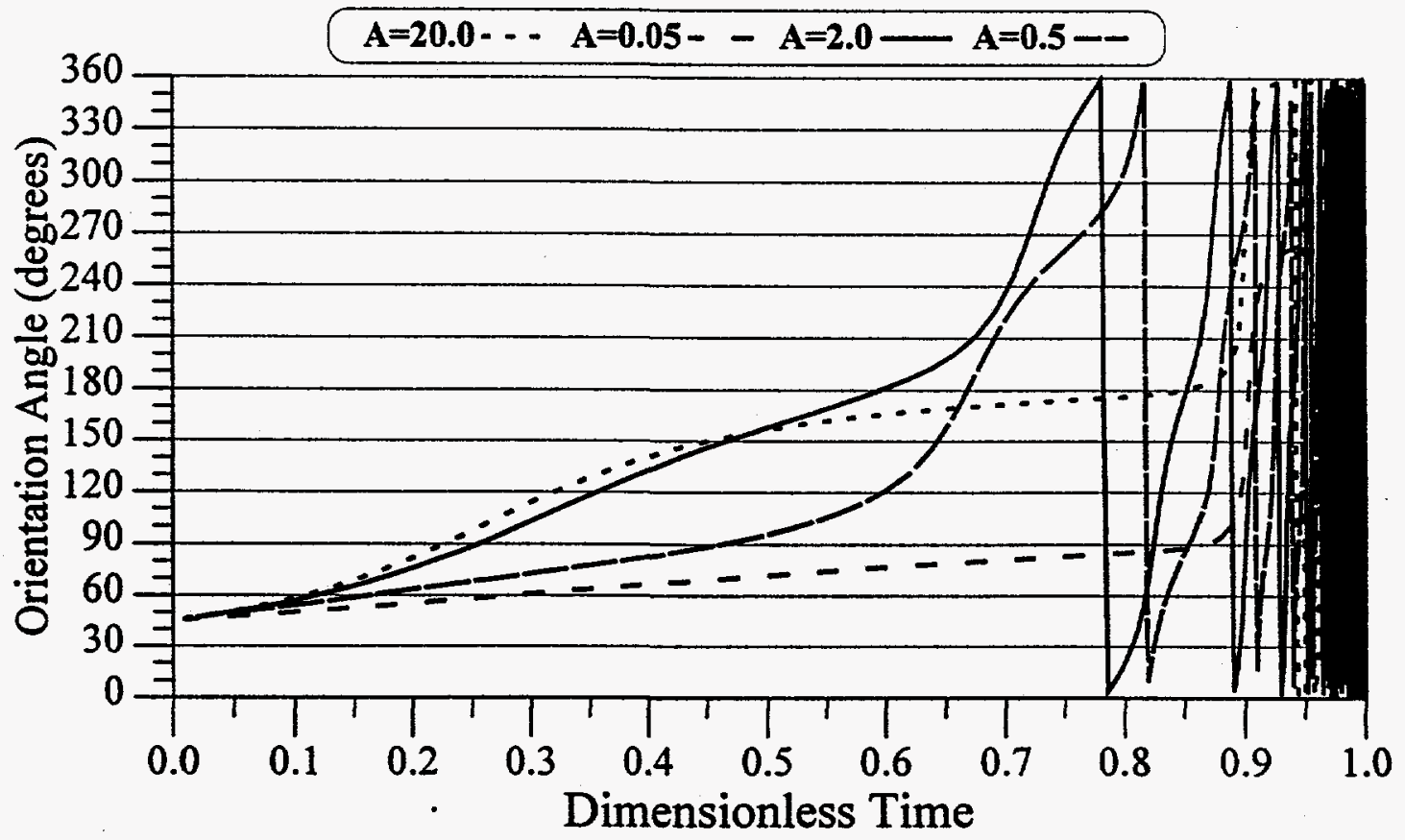

Figure 17 Comparison of oblate and prolate orientation evolution 
PSD Sensor Project including Shape Characterization

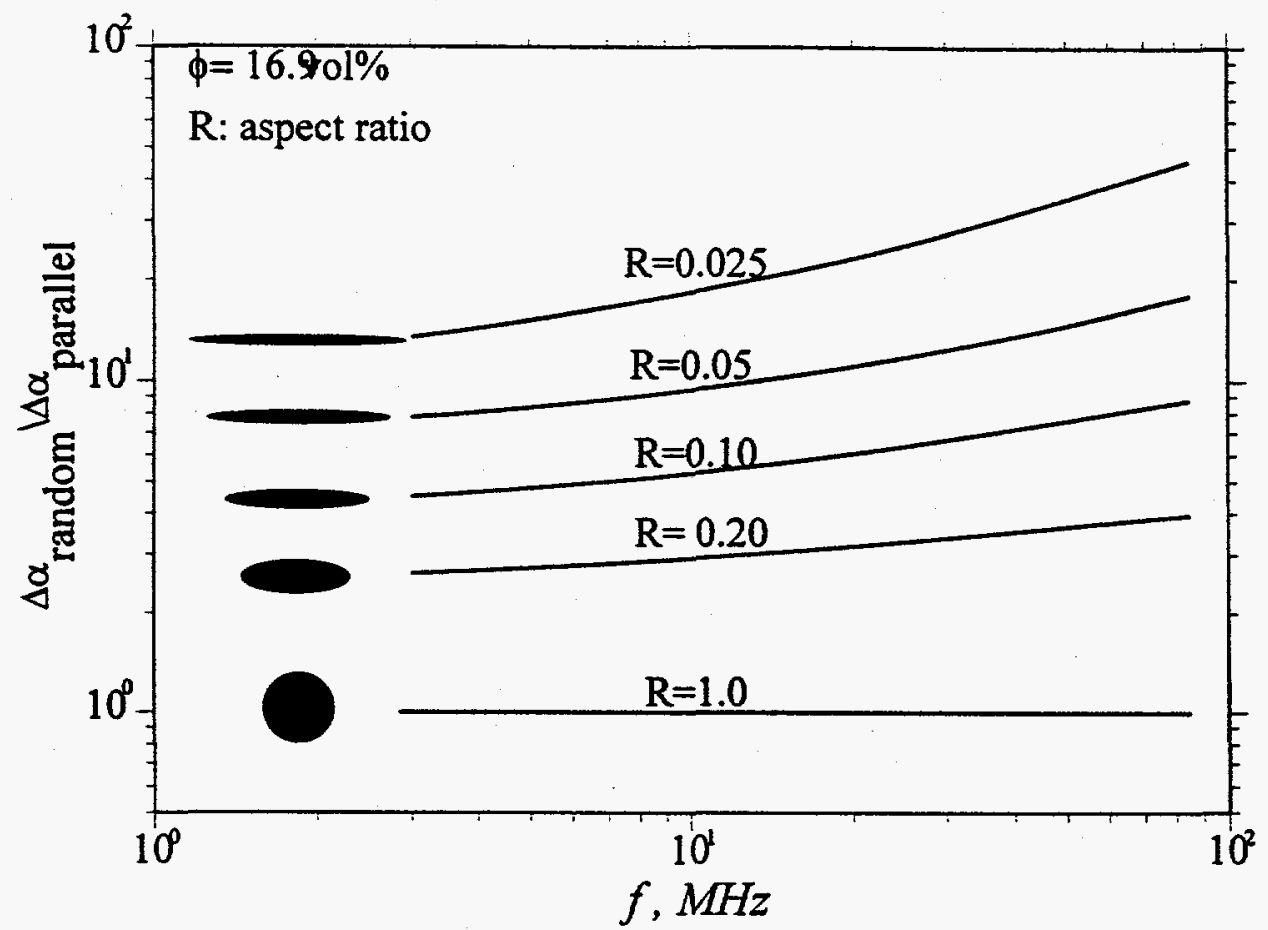

Figure $18 \mathrm{Model}$ predictions of effect of aspect ratio of clay particles on excess attenuation spectrum under random and parallel orientations. Particle size distributions with constant shape aspect ratio $R$ are used.

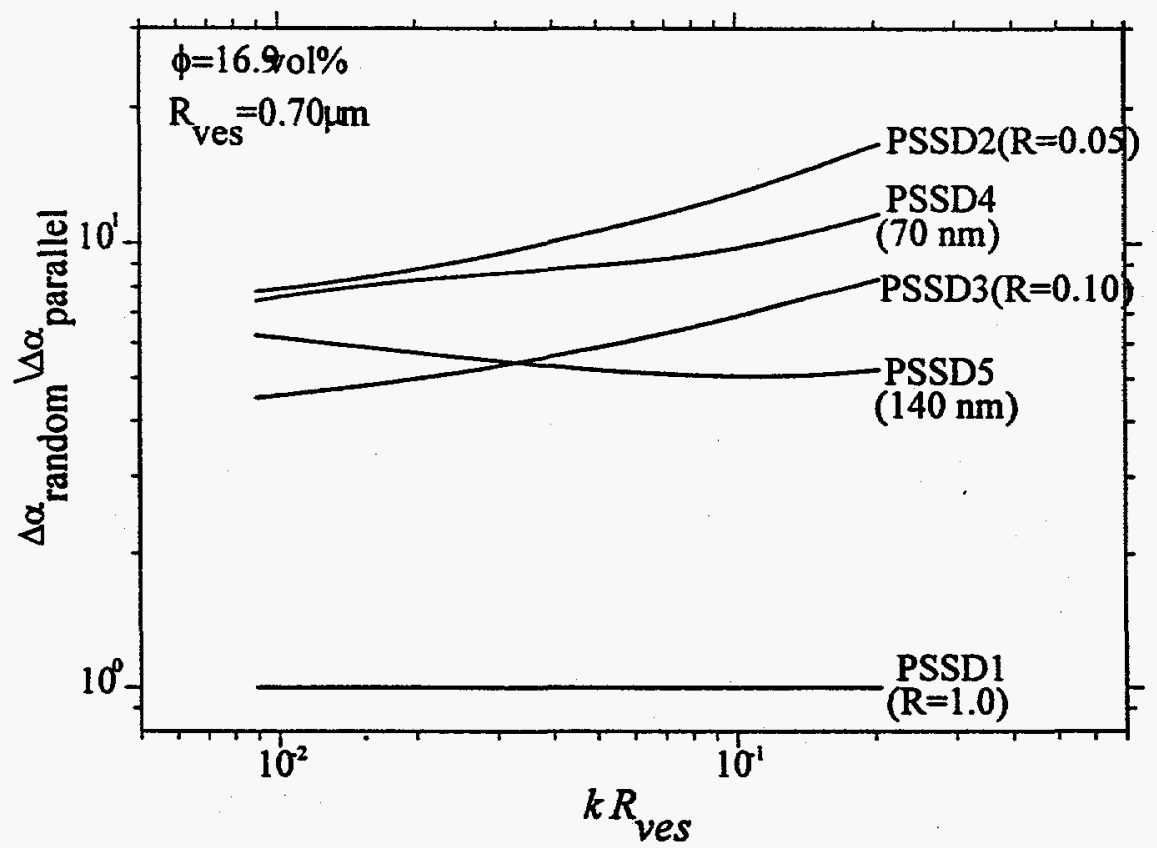

Figure 19 Model predictions of effect of aspect ratio of clay particles on excess attenuation spectrum under random and parallel orientations, based on five test Particle size and shape distributions. 
PSD Sensor Project including Shape Characterization

\section{List of Published Technical Papers resulting from the PSD Sensor project}

1. "Ultrasound-Based Sensors for Characterization of Colloidal Slurries", Pendse H. P. and Strout T. A., Sensors Expo West Proceedings, pp. 205B-1-7 (1990).

2. "Ultrasonic Sensor for the Characterization of Colloidal Slurries", Parker D., Lec R. M., Pendse H. P., and Vetelino J. F., IEEE Ultrasonics Symposium (1990).

3. "Theoretical Considerations in Acoustophoretic Analysis of Concentrated Colloids", Pendse H. P., Strout T. A. and Sharma A., in Electroacoustics for Characterization of Particulates and Suspensions, NIST Special Publication 856, (S. G. Malghan Editor), pp. 23-39 (1993)

4. "Particle Size Distribution Analysis of Industrial Colloidal Slurries using Ultrasonic Spectroscopy", Pendse H. P. and Sharma A., Particles and Particulate Systems Characterization, vol. 10, pp. 229-2333 (1993).

5. "On-line Instrumentation for Particle Size Distribution Analysis of Industrial Colloidal Slurries using Ultrasound", Pendse H. P. and Sharma A., Proceedings of First International Particle Technology Forum, Part 1, (AIChE), pp. 136-141 (1994).

6. "Ultrasound Characterization of Concentrated Colloidal Suspensions Containing Nonspherical Particles", Pendse H. P. and Han W., Proceedings of First International Particle Technology Forum, Part 1, (AIChE), pp. 142-147 (1994).

\section{List of Ph. D. Dissertations resulting from the PSD Sensor Project}

1. "Attenuation of Sound in High-Concentration Suspensions: Development and Application of an Oscillatory Cell Model", Terry A. Strout, Univeristy of Maine, Orono, ME (1991).

2. "Viscothermal Coupling Effects on Sound Attenuation in Concentrated Colloidal Disperstions", Wei Han, University of Maine, Orono, ME (1995).

3. "Experimental Aspects of Ultrasound Spectroscopy", Arvind Sharma, University of Maine, Orono, ME (1995).

(These $\mathrm{Ph}$. D. dissertations are available from UMI Dissertation Services, $300 \mathrm{~N}$. Zeeb Road, Ann Arbor, MI 48106 (1-800-521-0600)

\section{List of M. S. Theses resulting from the PSD Sensor Project}

1. "Application of uUltrasonic Spectroscopy to Characterize Papermaking Pulps: A Study of the Effect of Refining On the Acoustic Attenuation Response of Pulp Slurries", Diptarka Majumdar, M. S. Thesis, University of Maine, Orono, ME (1992).

2. "Particle Orientation Evolution in Suspensions Subjected to Squeezing Flows", Timothy C. Bliss, M. S. Thesis, University of Maine, Orono, ME (1996).

(These M. S. theses are available from the University of Maine Library, Orono, ME 04469) 
PSD Sensor Project including Shape Characterization

\section{Interim Technical Report on PSD Sensor Project (October 1992)}

(See Attached) 